\title{
Leben im Standby-Modus. Ergebnisse partizipativer Forschung zu den sozialen Folgen der Finanzkrise in Österreich
}

\author{
Ortrud Leßmann* und Elisabeth Buchner ${ }^{* *}$
}

\section{Zusammenfassung}

Dieser Beitrag beschreibt die sozialen Folgen der Finanz- und Wirtschaftskrise seit 2008 für ältere Arbeitssuchende in Salzburg auf der Grundlage der Ergebnisse eines EU-Projekts. Zentrale Frage ist, wie sich die Krise auf das Wohlergehen in Form der Rechte und Verwirklichungschancen im Leben der Betroffenen ausgewirkt hat und ob die Strategie der Sozialinvestitionen die Folgen abgemildert hat. Methodisch kombiniert das Projekt partizipative Aktionsforschung, Menschenrechte und Capability-Ansatz. Der Artikel ist die Verschriftlichung einer gemeinsam mit Betroffenen als Co-Forschenden erarbeiteten Präsentation der Ergebnisse, welche im Wechselspiel mit und als Folge von dem gesellschaftlichen Kontext dargestellt werden. Arbeitslosigkeit wirkt sich nicht nur auf den materiellen Lebensstandard aus, sondern beeinträchtigt das Wohlergehen auch in Bezug auf die Gesundheit, auf das familiäre und gesellschaftliche Leben und damit auf die Identität und Selbstentfaltungsmöglichkeiten - allesamt von den Menschenrechten geschützte Bereiche. Es ist nicht zu erkennen, inwiefern die österreichische Arbeitsmarktpolitik bei der Zielgruppe der Arbeitssuchenden über 45 Jahre „in Menschen investiert".

Schlagwörter: Well-being-Forschung, partizipative Aktionsforschung, Capability-Ansatz, Menschenrechte, ältere Arbeitslose in Österreich

\section{Living in standby-mode. Results of participatory research on the social consequences of the financial crisis in Austria}

\section{Abstract}

This article describes the social damage of the financial and economic crisis since 2008 on older unemployed persons in Salzburg, Austria. It is part of an EU-project evaluating the social investment strategy with the help of participatory methods. The article is based on the presentation of results jointly developed with unemployed who participated in the project. The research focused on the impact of the crisis on the well-being of vulnerable groups in terms of human rights and capabilities. The framework of participatory action research, human rights and capability approach is introduced briefly. Then the social context and the resulting impact on individual well-being are presented in turns. Unemployment does not only reduce the material standard of living, but also affects health, individual, family and social life and thus the identity and self-respect of the unemployed. These are all domains protected by human rights. The Austrian labor market policy does not consistently follow the social investment idea of "investing in people" with regard to the target group of older unemployed persons.

Keywords: well-being research, participatory action research, capability approach, human rights, unemployment of older persons in Austria

\footnotetext{
* Ortrud Leßmann, internationales forschungszentrum für soziale und ethische fragen. E-Mail: olessmann@ifz-salzburg.at ** Elisabeth Buchner, internationales forschungszentrum für soziale und ethische fragen. E-Mail: ebuchner@ifz-salzburg.at Wir möchten darauf hinweisen, dass die Co-Forschenden sowie einige Mitglieder der Steuerungsgruppe ganz im Sinne partizipativer Forschung über die Datengenerierung hinaus auch als Co-AutorInnen am Forschungsprozess beteiligt waren. Insofern ist dieser Beitrag eine Team-Leistung, wobei die Verantwortung für den Inhalt und mögliche Fehler allein bei den beiden Autorinnen verbleibt. Wir möchten uns herzlich für ihre aktive Teilnahme und die Bereitschaft, am Vortrag und Projektbericht mitzuwirken, bedanken.

This publication is part of the RE-InVEST project, this project has received funding from the European Union's Horizon 2020 research and innovation programme under Grant Agreement No 649447.
} 


\section{Einleitung}

In diesem Beitrag werden die Auswirkungen der Finanzund Wirtschaftskrise seit 2008 auf ältere Arbeitssuchende in Salzburg, Österreich, beschrieben. Er ist das Ergebnis des österreichischen Parts des europäischen Projekts RE-InVEST, das in zwölf europäischen Ländern untersucht, inwiefern es der Europäischen Kommission durch das Paket für Sozialinvestitionen von 2013 gelungen ist, durch Investitionen (in Menschen) die sozialen Folgen der Krise abzumildern. Die zentrale Fragestellung der ersten Projektphase war, wie sich die Finanz- und Wirtschaftskrise in individuellen Biografien besonders verletzlicher Personen hinsichtlich von Menschenrechten, Handlungsmöglichkeiten und Fähigkeiten (capabilities) ausgewirkt hat. Als theoretische Basis stützt sich das Projektkonsortium einerseits auf die Menschenrechte als einer schriftlich niedergelegten Wertebasis, welche die europäischen Länder jenseits aller kulturellen, ökonomischen und sozialen Unterschiede einigt, und andererseits auf den CapabilityAnsatz von Amartya Sen (2003; 2010) und Martha Nussbaum (Nussbaum 1999), dem es wesentlich darum geht zu definieren, was Wohlergehen (well-being) oder „Das gute Leben“ ist (siehe auch: Graf 2015). Sowohl die Menschenrechte wie auch der Capability-Ansatz weisen dabei darauf hin, dass das Leben - und damit auch das Wohlergehen der Menschen - viele Aspekte oder Dimensionen hat, die sich nicht einfach in einer Größe zusammenfassen lassen, sondern auch je für sich betrachtet und erfasst werden müssen. Sie trachten danach, diese Dimensionen des Wohlergehens zu benennen und gehören somit $\mathrm{zu}$ den Theorien, die das Wohlergehen einer Person vor allem an objektiven Bedingungen und weniger am subjektiven Wohlempfinden festmachen. Das Projekt ist somit ein Beispiel für Well-being-Forschung in Österreich.

Der gewählte theoretische Rahmen legt zugleich die gewählte Methode nahe: Was zum guten Leben gehört, steht nicht ein für alle Mal fest, sondern es sind nach Sen (2010, Kap. 11) jene Dinge, die eine Person aus guten Gründen wertschätzt. Daher sind diejenigen einzubeziehen, um deren Leben es geht, und danach zu befragen, was ihres Erachtens ein gutes Leben ausmacht. Der Katalog der Menschenrechte - einschließlich der sozialen und ökonomischen Menschenrechte - eignet sich zur Orientierung, muss jedoch für den jeweiligen Kontext spezifiziert werden. Letztlich will Sen dem „öffentlichen Vernunftgebrauch“ (public reasoning) nicht vorgreifen und hat daher keine Liste der Elemente eines guten Lebens erstellt. Aus diesem Grund wurde ein partizipativer Forschungsansatz gewählt. Im Rahmen von RE-InVEST wurde eine geeignete Methodik entwickelt (PAHRCA - Participatory Action Human Rights and Capability Approach), die den theoretischen Rahmen in einen partizipativen, qualitativen Forschungsprozess integriert.

Dieser Beitrag erläutert zunächst in Abschnitt 2 den theoretischen und methodischen Rahmen des Projekts RE-InVEST und seine Umsetzung im österreichischen Teilprojekt. Die Ergebnisse des partizipativen Forschungsprozesses in Österreich werden in Abschnitt 3 dargestellt. Abschnitt 4 stellt zum Schluss die Frage, ob für die Zugehörigkeit in der österreichischen Gesellschaft wirklich nur die Arbeit zählen soll.

\section{Theoretischer und methodischer Rahmen}

Der theoretische und methodische Rahmen für die Forschung mit Betroffenen in Salzburg wurde zum Großteil vom europäischen Projektkonsortium gemeinsam festgelegt und vor Ort in Absprache mit den lokalen Projektteilnehmerinnen und -teilnehmern angepasst. Die Verbindung von Menschenrechten und Capability-Ansatz zu einem theoretischen Rahmen ${ }^{1}$ sowie die Entscheidung, Methoden der partizipativen Aktionsforschung anzuwenden, sind dabei auf der Ebene des Gesamtprojekts gefallen. Die Auswahl der konkreten Methoden verblieb jedoch bei den Projektbeteiligten in Salzburg.

Die Menschenrechte bilden in Europa eine gemeinsame Wertebasis und können auch als Auflistung von Elementen, die ein gutes Leben ausmachen, verstanden werden. Es sind Grundrechte und Freiheiten, die jeder und jedem zustehen. Zugleich verleihen ihnen internationale Gesetzgebung einschließlich einiger Vereinbarungen und Konventionen rechtliche Gültigkeit. Die Vorstellung von grundlegenden Menschenrechten hat sich über viele Jahrhunderte hinweg entwickelt und nach dem Zweiten Weltkrieg breite Unterstützung gewonnen, als die Vereinten Nationen 1948 die Allgemeine Erklärung der Menschenrechte verabschiedet haben. In dieser Erklärung wird erstmals aufgeführt, welche Menschenrechte und grundlegenden Freiheiten allen Menschen ohne jegliche Diskriminierung zustehen. Menschenrechte definieren einen allgemein

1 Grundlegende Literatur zur Kombination von Capability-Ansatz und Menschenrechten: Elson, 2014; Nussbaum, 2002; Sen, 2005. 
Abbildung 1: Ressourcen, Umwandlungsfaktoren und Verwirklichungschancen

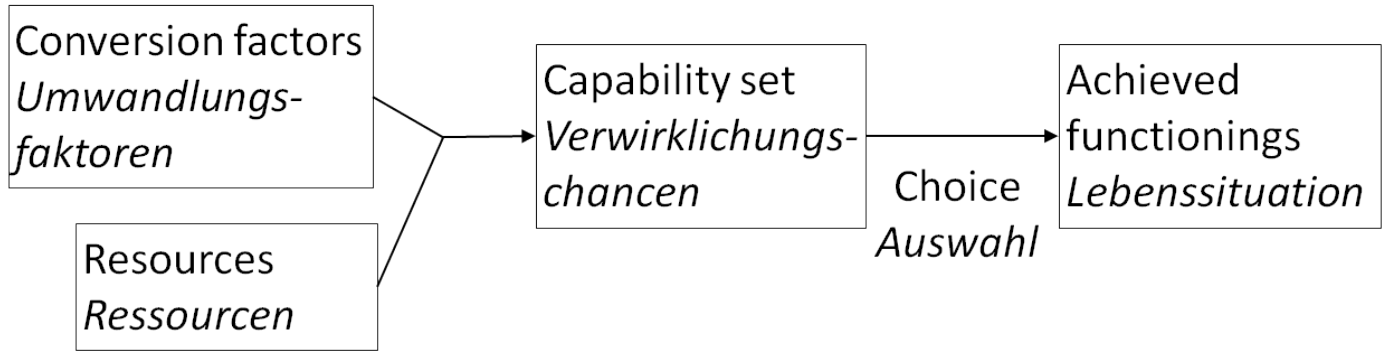

Quelle: eigene Darstellung, in Anlehnung an Robeyns (2005: 98)

akzeptierten Standard, der sicherstellen soll, dass die Würde jeder Person gewahrt und ihr mit Achtung begegnet wird. Die Menschenrechte sind unabhängig voneinander einzuhalten und unteilbar, d. h. sie sind miteinander verbunden und der Verstoß gegen ein Menschenrecht kann Auswirkungen auf die Wahrung der anderen haben. In der Regel durch gesetzliche Bestimmungen - internationale und regionale Abkommen oder nationale Gesetzgebung - schriftlich festgehalten, treffen die Menschenrechte eine Aussage über allgemein akzeptierte Prinzipen darüber, wie ein Staat seine Bürgerinnen und Bürger und andere Menschen, die innerhalb seiner Grenzen leben, behandeln soll. Sie beinhalten sowohl bürgerliche und politische Rechte - unter anderem das Recht auf Leben, das Recht auf Gleichheit vor dem Gesetz und das Recht, keiner Folter ausgesetzt zu werden - als auch ökonomische, soziale und kulturelle Rechte wie das Recht auf Arbeit, das Recht, einer Gewerkschaft beizutreten, das Recht auf Gesundheit, Bildung und einen angemessenen Lebensstandard. Einige besondere Gruppen wie Frauen, Kinder, Menschen mit Behinderungen, Minderheiten sowie Migrantinnen und Migranten werden durch spezielle Vereinbarungen geschützt. Für benachteiligte Menschen hat die Benennung von „Rechten“ eine stärkende Wirkung. Sie haben mehr Selbstbewusstsein, das einzufordern, was ihnen in ihrem Leben fehlt, wenn es als ihr Recht anerkannt ist.

Nach dem Capability-Ansatz von Sen ist für ein gutes Leben zum einen die Lebenssituation wichtig was ein Mensch tut oder ist (achieved functionings) - und zum anderen sind die Verwirklichungschancen wichtig - was ein Mensch zu tun oder zu sein in der Lage ist (capabilities). Um ein gutes Leben in diesem Sinne führen zu können, braucht eine Person sowohl (materielle) Ressourcen als auch andere Voraussetzungen (Umwandlungsfaktoren genannt), die persönlicher oder gesellschaftlicher Natur sind oder von den Gege- benheiten der Umwelt abhängen (Abb. 1). Ressourcen werden dabei verstanden als Güter und Dienstleistungen, die eine Person zur Gestaltung ihres Lebens nutzt. Beispiele persönlicher Umwandlungsfaktoren sind (erworbene) Fähigkeiten, aber auch körperliche Voraussetzungen. Bei den gesellschaftlichen Umwandlungsfaktoren lässt sich zwischen sozialen Normen einerseits und institutionellen Regelungen andererseits unterscheiden. Mit Umweltfaktoren sind schließlich geografische Gegebenheiten (Berge, Flüsse, Täler), das Klima, aber auch Umweltbelastungen gemeint. Entscheidend ist nach Sen, ob all diese Voraussetzungen einer Person ein Leben ermöglichen, das sie aus guten Gründen wertschätzt. Es geht also nicht nur um ihre tatsächlich erreichte Lebenssituation, sondern auch um ihre Entscheidungsfreiheit bzw. ihre Möglichkeiten, ihr Leben selbst zu gestalten (ihre Verwirklichungschancen).

Um einschätzen zu können, wie es um die Verwirklichungschancen von verletzlichen Personengruppen steht, werden bei der qualitativen Forschung im Projekt RE-InVEST deren Erfahrungen durch einen partizipativen Forschungsansatz ins Zentrum gerückt.

„Partizipative Forschungsmethoden sind auf die

Planung und Durchführung eines Untersuchungsprozesses gemeinsam mit jenen Menschen gerichtet, deren soziale Welt und sinnhaftes Handeln als lebensweltlich situierte Lebens- und Arbeitspraxis untersucht wird. In der Konsequenz bedeutet dies, dass sich Erkenntnisinteresse und Forschungsfragen aus der Konvergenz zweier Perspektiven, $d$. h. vonseiten der Wissenschaft und der Praxis, entwickeln. Der Forschungsprozess wird im besten Falle zum Gewinn für beide Seiten" (Bergold/Thomas 2012).

Partizipative Aktionsforschung stellt eine Variante dieses Forschungsansatzes dar und bedeutet, dass die Betroffenen gemeinsam und auf Augenhöhe mit den Forschenden an einem partizipativen Prozess von Wissenserzeugung, Reflexion und Aktion teilnehmen. 


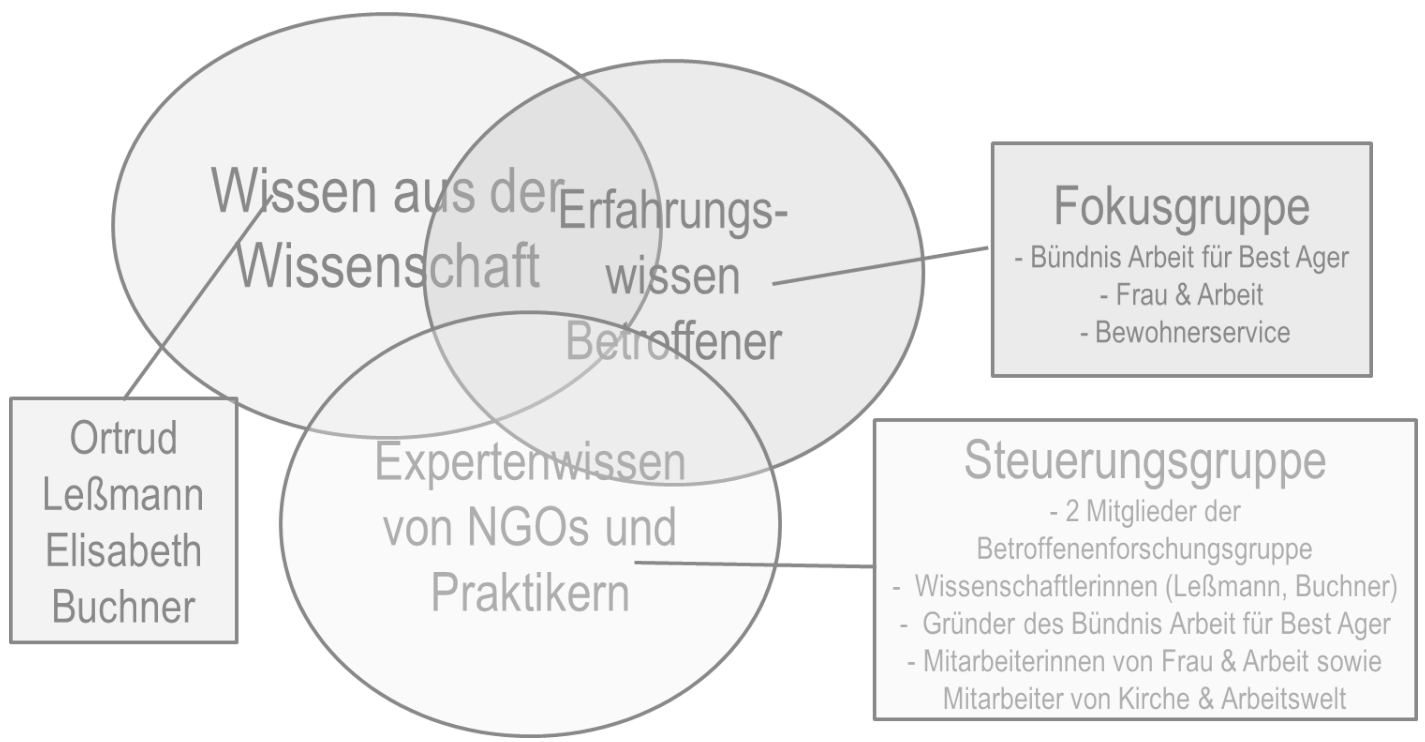

Quelle: eigene Darstellung

Partizipative Aktionsforschung folgt einem zyklischen Prozess von Wissensgenerierung, Stärkung der Betroffenen durch Teilhabe und Empowerment sowie einer Aktion, die dieses Wissen nutzt, um die soziale Wirklichkeit zu verändern.

Die Lebenswelten und Sinndeutungen der Betroffenen wurden durch verschiedene Varianten autobiografisch-narrativer Gruppeninterviews (Biografieschlange, Erzählkreis) erhoben. Diese Daten bildeten die Grundlage für die eigentliche Wissensgenerierung durch die Methoden "Merging of Knowledge“ (Abb. 2). Damit wird die Verschränkung verschiedener Wissensbestände mit dem Ziel eines tieferen, facettenreichen Problemverständnisses - und damit verbunden der Sicherung von Güte und Qualität - bezeichnet. Im mehrmonatigen Forschungsprozess fanden in Salzburg vier Workshops der Betroffenenforschungsgruppe und zwei Treffen der Steuerungsgruppe - bestehend aus sieben lokalen Expertinnen und Experten der Praxis, die mit der Zielgruppe beruflich arbeiten, zwei Betroffenen und den wissenschaftlichen Projektmitarbeiterinnen - statt. Indem die generierten Wissensbestände in jeder Phase in alle Gruppen eingespeist wurden, konnten diese kontextualisiert, spezifiziert und somit partizipativ validiert werden.

Als einheitlicher Rahmen wurde für das Projekt RE-InVEST ein Forschungsablauf festgelegt, der Menschenrechte, Capability-Ansatz und partizipative Aktionsforschung integriert: PAHRCA (Murphy/Hearne,
2015). ${ }^{2}$ Dieser sieht sieben Schritte vor (Murphy/Hearne 2015: 44-45): 1. Ermittlung einer geeigneten PartnerNGO; 2. Kontaktaufnahme und erste Treffen mit der NGO zwecks Vertrauensbildung; 3. Erstes Treffen mit den Teilnehmerinnen und Teilnehmern - Vertrauensbildung, Festlegung des Forschungsablaufs; 4. Entwicklung eines gemeinsamen theoretischen Verständnisses auf der Grundlage von Menschenrechten und Capability-Ansatz; 5. Forschungsphase und Datenerhebung; 6. Analyse und gemeinsame Identifikation von Kernthemen und -belangen der Gruppe und 7. Durchführung einer Aktion als Ergebnis des Forschungsprozesses.

Der erste Schritt bestand darin, festzulegen, welche Zielgruppe in Salzburg für das Forschungsvorhaben

2 Kernprinzipien dieses Ansatzes (Murphy/Hearne 2015: 40): (1) Positive discrimination in the allocation of time and resources, with priority being given to the weakest participants in the process. (2) Effective investment in the research capacity \& capability of those groups. (3) Phasing in of joint collaboration to the highest possible levels. (4) Adaptation of analytical instruments and language. (5) Interculturally (\& gender, age, etc.) sensitive approaches, including mediation and training of all participants. Specific attention will be devoted to the composition of our 'mixed research teams', particularly also the gender/age/ethnic profile of participants from vulnerable groups. (6) Continuity and feedback at all stages of the research. (7) Empowering (8) Dialogical and reflexive approaches. (9) Communicative and democratic decision-making. 
geeignet ist. ${ }^{3}$ Neben der besonderen Verletzlichkeit und Betroffenheit durch die Krise als zentralem Kriterium beeinflussten auch pragmatische Überlegungen hinsichtlich des Zugangs zum Feld und der Möglichkeiten für eine langfristige, partizipative Zusammenarbeit die Auswahl. Weil die Erwerbsarbeitslosigkeit ${ }^{4}$ in Österreich insbesondere bei Menschen ab ca. 45 Jahren seit einigen Jahren deutlich steigt und diese vermehrt von verfestigter Arbeitslosigkeit betroffen sind, hat das Institut in Salzburg gezielt einen Praxispartner aus diesem Bereich gesucht und mit dem Bündnis Arbeit für Best Ager (BABA) auch gefunden (Schritt 1). Das Bündnis Arbeit für Best Ager ist auf Initiative einiger Betroffener entstanden und versteht sich als Lobbygruppe für die Belange Älterer (45-65 Jahre) in puncto Beteiligung am Erwerbsleben. Bei einem Vortreffen (Schritt 2) zwischen den Wissenschaftlerinnen des Instituts mit dem Leitungsgremium des Bündnis Arbeit für Best Ager wurde die Zusammenarbeit in dem Forschungsprojekt fixiert und zugleich vereinbart, auch Frau \& Arbeit (Frauenservicestelle für berufliche Themen, gefördert vom Land Salzburg, dem Arbeitsmarktservice Salzburg und anderen), Kirche und Arbeitswelt (getragen von der katholischen Kirche) und die Bewohnerservicestellen der Stadt Salzburg zur Mitarbeit einzuladen. Über diese Partner fand sich (durch Aushänge in den Institutionen und E-Mail-Aussendungen) eine Gruppe aus neun erwerbsarbeitslosen Menschen ab 45 Jahren, die sich freiwillig bereit erklärten, langfristig als Co-Forschende am Projekt teilzunehmen. Angestrebt wurde größtmögliche Heterogenität der Teilnehmenden hinsichtlich soziodemografischer Merkmale und dem Vorliegen bzw. der Kombination von aus der Forschung bekannten Risikofaktoren, die eine besondere Verletzlichkeit der Betroffenen hinsichtlich der Arbeitsmarktintegration wahrscheinlich machen. ${ }^{5}$ Diese Zielsetzung

3 Die Zielgruppen in den anderen Partnerländern reichten von Alleinerziehenden, Migrantinnen und Migranten (mit und ohne gültigen Aufenthaltsstatus), Menschen mit Behinderungen, wohnungslosen bis zu verschiedenen Gruppen arbeitsloser Menschen (Jugendliche, langzeitarbeitslose Menschen, ältere Arbeitslose).

4 Die Termini „Arbeitslosigkeit“ und „Erwerbsarbeitslosigkeit" werden in diesem Beitrag synonym verwendet.

5 Hier sind insbesondere zu nennen: die Kombination aus Migrationshintergrund und geringer Qualifikation, gesundheitliche Beeinträchtigungen/Behinderung, (Allein-) Erziehungs- oder Pflegeverpflichtungen, prekäre Beschäftigungsbiografien, Langzeitarbeitslosigkeit/Langzeitbeschäftigungslosigkeit steht in einem gewissen Spannungsverhältnis zur Methodik der partizipativen Aktionsforschung, die die Fähigkeit und den Willen zu einer längerfristigen, freiwilligen Partizipation und damit gewisse Ressourcen bei den Teilnehmenden voraussetzt. Die Heterogenität des Samples konnte somit nur partiell erreicht werden. ${ }^{6}$

Die vier Workshops dauerten je knapp vier Stunden und fanden in einem Zeitraum von vier Monaten (Nov. 2015 bis Feb. 2016) statt. Im ersten standen die Vorstellung des Projekts sowie das gegenseitige Kennenlernen und die Schaffung einer vertrauensvollen Atmosphäre im Vordergrund (Schritt 3). Insbesondere wurden die theoretischen Bezüge, der zeitliche Rahmen sowie die Methoden des Projekts erläutert. Die Beteiligten erarbeiteten einige Regeln für die Zusammenarbeit in der Gruppe.

Im zweiten Workshop (Schritt 4 und 5) stand die Arbeit an der individuellen Biografieschlange (Murphy/Hearne 2015: 49) im Mittelpunkt, bei der die Teilnehmenden gebeten wurden, die wichtigsten Ereignisse in den vergangenen zehn Jahren ihres Lebens chronologisch geordnet $\mathrm{zu}$ benennen und im Anschluss in Kleingruppen zu erzählen. Die Zeitleiste in Form einer Schlange fungierte als Erzählanreiz für ein narratives Gruppeninterview. Die Schlange erlaubt es, Aufwärts- und Abwärts-Entwicklungen in der persönlichen Biografie zu verorten. Die persönliche „Krisengeschichte" wurde so erhoben.

Im zweiten Teil des Workshops wurde an einem gemeinsamen Verständnis des theoretischen Rahmens gearbeitet. Die Teilnehmenden wurden aufgefordert, jeweils die drei für sie selbst wichtigsten Elemente eines guten Lebens auf eine Karte zu schreiben und der Gruppe zu erläutern, warum sie diese Elemente so

6 Beschreibung des Samples: sechs Frauen, drei Männer, alle längere Zeit auf Arbeitssuche, fünf davon langzeitbeschäftigungslos (Definition siehe Fußnote 9), vier haben einen anderen Status (längerer Krankenstand, Unterbrechung einer längeren Arbeitslosigkeit durch mehrmonatige Beschäftigung), sechs Personen aus Salzburg Stadt, drei aus kleineren Städten im Umland, eine Person mit Migrationshintergrund (Deutschland), alle verfügen über mindestens mittleres Bildungsniveau (Lehre oder Matura, eine Person hat kürzlich ein Studium abgeschlossen), drei haben starke psychische Belastungen durch Arbeitsbedingungen in vergangenen Beschäftigungsverhältnissen erlebt, drei haben psychische Belastungssymptome im Zeitraum der Arbeitslosigkeit entwickelt, zwei Personen mit Pflegeverpflichtungen, zwei Personen mit längeren Beschäftigungsphasen bei Leiharbeitsfirmen, zwei Personen waren längere Zeit (prekär) selbstständig beschäftigt. 
wichtig finden. Die entstandene Liste wurde dann der Liste gegenübergestellt, die Burchardt und Vizard (2011: 101) auf der Grundlage von Capability-Ansatz und Menschenrechten entwickelt haben. ${ }^{7}$ Die Teilnehmenden betonten vor allem folgende Dimensionen aus dieser Liste: Recht auf (1) Gesundheit und (2) angemessenen Lebensstandard, aber auch (5) individuelles, Familienund gesellschaftliches Leben (Freunde, Familie, soziale Kontakte) sowie (4) Identität, Selbstentfaltung und Selbstachtung. Ferner fanden sich in ihren Antworten Anklänge an das Recht auf (3) Bildung und Lernen, (6) Teilhabe und Mitsprache, (7) Rechtssicherheit und (8) produktive wertgeschätzte Tätigkeit.

Im dritten Workshop (Schritt 5) wurde der Anschluss zu dieser Übung hergestellt, indem die Teilnehmenden gebeten wurden, einzuschätzen, inwiefern sie über die gelisteten Elemente eines guten Lebens im Moment verfügen und ob sie für die Zukunft eine Verbesserung, Beibehaltung oder Verschlechterung ihrer Situation hinsichtlich dieser Dimensionen erwarten. Im Anschluss daran wurde die "große Schlange“ (Murphy/Hearne 2015: 62) gemeinsam erarbeitet, bei der die gesellschaftlichen, wirtschaftlichen und politischen Entwicklungen der letzten zehn Jahre in Salzburg, Österreich und der EU benannt und festgehalten wurden. Die Teilnehmenden wurden angeregt, darüber nachzudenken, wo ihre individuellen „Krisenbiografien" mit den krisenhaften Entwicklungen auf kollektiver Ebene verbunden oder auch unverbunden sind und welche Auswirkungen auf Menschenrechte und Capabilities erkennbar sind. Schließlich wurde als zentrale Methode der Datenerhebung ein biografischer Erzählkreis durchgeführt, bei dem drei Betroffene sich bereit erklärten, ihre individuelle „Krisenbiografie“ der letzten Jahre detailliert zu erzählen. Die anderen Gruppenmitglieder hörten zu, beobachteten und hatten im Anschluss die Möglichkeit, die Erzählungen zu ergänzen und ihre Beobachtungen mitzuteilen. Die so erhobenen Daten bildeten die Grundlage für zwei biografische Einzelfallstudien für den Projektbericht.

In einem vierten Workshop wurden die von den Projektleiterinnen zusammengefassten Ergebnisse diskutiert und gemeinsam Muster und Kernkategorien festgelegt (Schritt 6). Insbesondere ging es darum, gemeinsame Deutungen hinsichtlich der Auswirkun-

$7 \quad$ Wobei die Dimensionen Recht auf Leben, körperliche Unversehrtheit und Gesundheit zu einer Dimension "Gesundheit" zusammengefasst wurden, sodass die genannten acht Dimensionen blieben. gen der Krise auf „Menschen wie sie“ auf Basis des im Rahmen des Projekts neu generierten Wissens herauszuarbeiten. Ferner hat die Gruppe hinsichtlich des bestehenden Handlungsbedarfs als wichtigstes Thema eine Veränderung der aktiven Arbeitsmarktpolitik identifiziert und darüber beraten, welche Aktion sie auf der Grundlage der Ergebnisse durchführen möchte (Schritt 7). Die Erhöhung der Sichtbarkeit der Thematik und der betroffenen Personen in der Öffentlichkeit wurde als notwendiger Schritt für die Ziele der Sensibilisierung von Gesellschaft und Politik sowie die Veränderung der aktiven Arbeitsmarktpolitik definiert. Die Projektbeteiligten haben deshalb gemeinsam eine Präsentation erarbeitet und die Projektergebnisse im Rahmen einer wissenschaftlichen Fachtagung zur Zukunft der sozialen Sicherung vorgetragen. Dieser Beitrag ist die schriftliche Ausarbeitung dieses Vortrags. Ferner haben sie einen Zeitungsartikel initiiert und die Ergebnisse im Rahmen einer Sendung eines Lokalradios sowie in einem Erzählcafé bei der Salzburger Armutskonferenz 2016 vorgestellt.

Alle Treffen wurden von den ifz-Wissenschaftlerinnen detailliert protokolliert. Die Protokolle wurden den Teilnehmenden für Ergänzungen und Korrekturen zeitnah übermittelt. Einzelne Erhebungsphasen der Betroffenen-Workshops (individuelle Schlange, Erzählkreis) wurden auf Tonband aufgezeichnet und transkribiert. Die Auswertung erfolgte in Orientierung an die qualitative Inhaltsanalyse nach Mayring (1993). Die Kernkategorien wurden mit der Betroffenenforschungsgruppe auf Basis des so vorstrukturierten Materials festgelegt.

Die Steuerungsgruppe lieferte das lokale „Expertenwissen“ im Rahmen des Merging-of-KnowledgeProzesses. Sie traf sich zwei Mal zwischen den Betroffenen-Workshops, um die Ergebnisse zu diskutieren und durch ihr spezifisches Hintergrundwissen zu vertiefen.

\section{Die Krise als schleichender Prozess}

Wie oben beschrieben, wurde das Sinnbild der Schlange einerseits verwendet, um die Teilnehmenden über ihr persönliches Leben in den letzten zehn Jahren nachdenken zu lassen, und andererseits, um sich gemeinsam die politischen, wirtschaftlichen und sozialen Ereignisse auf lokaler, nationaler und europäischer Ebene in Erinnerung zu rufen und so das eigene Erleben dazu in Kontext zu setzen. Besser als ein gerader Zeitstrahl vermag das Bild der Schlange auszudrü- 
Abbildung 3: Die Schlange - Symbol der schleichenden Krise

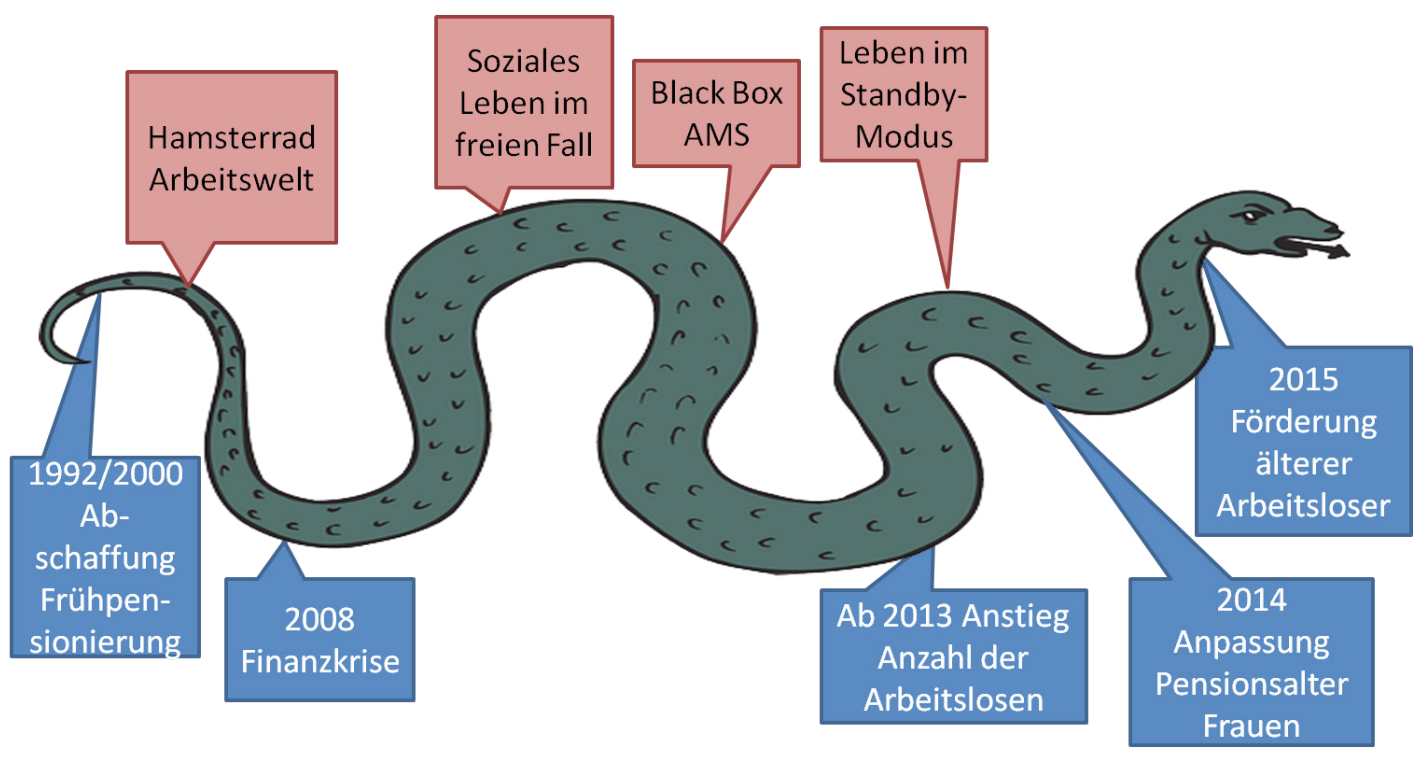

Quelle: eigene Darstellung

cken, dass nicht alle Ereignisse gleich wichtig sind und sich nicht immer eindeutig einem Zeitpunkt zuordnen lassen, sondern dass das Leben Höhen und Tiefen hat. Die Vorstellung, dass sich das Leben „dahinschlängelt“ spricht die Intuition an und wurde von den Teilnehmenden so geschätzt, dass die Gruppe beschloss, auch das Ergebnis der Forschung anhand dieses Bildes darzustellen, zumal die Schlange gut zu einem Kernergebnis des Forschungsprozesses passt (Abb. 3): Die Finanzkrise von 2008 wurde in Österreich, anders als in vielen anderen EU-Ländern, nämlich nicht als herausragendes Ereignis wahrgenommen, sondern eher als Teil eines schleichenden Prozesses, der bereits Jahre zuvor eingesetzt hat. Die unteren, blauen Einträge in Abbildung 3 beziehen sich dabei auf den politischen und wirtschaftlichen Kontext (Abschnitte 3.1, 3.3 und 3.7), wie er von der Gruppe in der "großen Schlange“ (Abschnitt 2) geschildert und dann mit Hilfe von Literaturstudien verdichtet wurde. Die oberen, roten Einträge stellen die Kernergebnisse der qualitativen Forschung (Abschnitte 3.2, 3.4-3.6) vor. Die Themen sind grob nach ihrer zeitlichen Relevanz geordnet: Dass die politischen Reformen des Pensionsantritts (3.1) nur zögerlich umgesetzt wurden, fiel in Zeiten des Wirtschaftswachstums nicht auf, während gleichzeitig der Druck auf die Beschäftigten stieg (Hamsterrad 3.2). Die Finanzkrise wurde in Österreich zunächst gut aufgefangen, doch die Analyse zeigt, dass im Nachgang ältere Beschäftigte besonders von Arbeitslosigkeit betroffen sind, da präventive und investive Maßnahmen fehlten (3.3). Die Betroffenen erleben einerseits, dass sich die Basis ihres Wohlergehens rapide verschlechtert (3.4), und müssen sich andererseits mit dem Arbeitsmarktservice in einer „Bittstellerposition“ auseinandersetzen (3.5). Sie leben im „Standby-Modus“ (3.6), der ihr Wohlergehen in vielerlei Hinsicht beeinträchtigt. Umfassende Maßnahmen zur Beschäftigung Älterer (3.7) wurden erst eingeführt, als das Projekt bereits läuft.

Im Folgenden wird einerseits die Krise als schleichender Prozess auf der gesellschaftlichen Ebene nachgezeichnet und andererseits geschildert, wie die Teilnehmenden ihre daraus resultierende Arbeitslosigkeit auf individueller Ebene erlebt haben.

\subsection{Kontext: Politische Reformen des Pensions- antrittsalters}

Das reguläre Pensionsantrittsalter liegt in Österreich für Männer bei 65 und für Frauen bei 60 Jahren, wobei letzteres seit 2014 schrittweise an jenes der Männer nach oben angepasst wird. Das tatsächliche Pensionsantrittsalter liegt jedoch mit 61,9 Jahren bei Männern deutlich darunter und ist auch bedeutend niedriger als der OECD-Durchschnitt von 64,2 Jahren (bei Frauen lagen die Werte bei 59,4 im Vergleich zum OECD-Durchschnitt von 63,1 Jahren, vgl. Hanappi/ Müllbacher/Heil 2014). Traditionell war der vorzeitige Pensionsantritt ein in Österreich weit verbreitetes Mittel der Arbeitsmarktpolitik, um Arbeitsmarktproblemen zu begegnen. Als Folge sind die Ausgaben für vorzeitige Alterspensionierungen bis zur Jahrtausend- 
wende stetig und stark gewachsen (BMASK 2014: 227). Durch mehrere Pensionsreformen ab 1992 und speziell nach der Jahrtausendwende wurden die Möglichkeiten für einen vorzeitigen Pensionsantritt deutlich reduziert und der Zugang zur Invaliditätspension wurde erschwert. Dennoch lag die Beschäftigungsquote der Personen im Alter zwischen 55 und 64 noch 2008, trotz der bis dahin guten Wirtschaftsentwicklung, bei nur 41 \%. Im Jahr 2015 lag sie bei 45,1 \% und damit im EUVergleich noch immer auf sehr niedrigem Niveau (Statistik Austria 2016). Auch als Folge dieser langjährigen Praxis ist der österreichische Arbeitsmarkt aktuell nur schlecht auf die längere Erwerbsbeteiligung und besonders die (Re-)Integration älterer Arbeitnehmerinnen und Arbeitnehmer eingestellt. Ältere Erwerbspersonen (45 Jahre oder älter) sind zwar meist stabil beschäftigt und werden seltener arbeitslos als jüngere. Wenn sie ihren Arbeitsplatz verlieren, haben sie jedoch viel größere Schwierigkeiten, eine neue Beschäftigung zu finden. Deshalb sind sie im Durchschnitt länger erwerbsarbeitslos und machen einen größeren Anteil der Langzeitbeschäftigungslosen ${ }^{8}$ (über ein Drittel) sowie fast die Hälfte der Langzeitarbeitslosen ${ }^{9}$ aus (Arbeitsmarktservice Österreich 2015a). Es kommt bei dieser Gruppe also vermehrt zu einer Verfestigung von Erwerbsarbeitslosigkeit und damit verbunden einem erhöhten Verarmungsrisiko.

\subsection{Ergebnisse: Hamsterrad Arbeitswelt}

„Es ist nur noch eine Zitrone auspressen bis zum Geht-Nicht-Mehr. (...) Du hast jedes Jahr mehr und mehr Arbeit, noch mehr Reisen, noch mehr Rennereien, noch mehr Druck (...).“(L.)

Die Projekt-Teilnehmenden legten als einen Kernbegriff, der ihre Biografien verbindet, jenen vom "Hamsterrad Arbeitswelt" fest. Dieser bringt ihre Erfahrungen vor Eintritt der Erwerbsarbeitslosigkeit auf den Punkt und verdeutlicht die Beeinträchtigung des Rechts (8) auf eine produktive, wertgeschätzte Tätigkeit. Die Wahrnehmung eines rasanten Wandels der

8 Als langzeitbeschäftigungslos gelten laut AMSDefinition Personen, die über ein Jahr arbeitslos vorgemerkt sind, wobei Schulungszeiten und bis zu zweimonatige Phasen der Erwerbstätigkeit diese Meldung nicht unterbrechen. (Arbeitsmarktservice Österreich 2015b)

9 Laut AMS-Definition zählen dazu Personen, die „über ein Jahr durchgehend und ohne längere Unterbrechung (auch nicht durch Schulung) arbeitslos vorgemerkt" sind. (Arbeitsmarktservice Österreich 2015b)
Arbeitswelt hinsichtlich Arbeitsbedingungen, Qualifikationsanforderungen sowie Konventionen und Kultur in Richtung einer Zunahme von Tempo, Wettbewerb und Unsicherheit findet sich auch in den Beiträgen der Steuerungsgruppenmitglieder wieder.

M. berichtet, dass inzwischen - besonders im Leiharbeitsbereich - vorausgesetzt wird, dass die volle Leistung quasi aus dem Stand erreicht wird, während noch in den 1990er-Jahren eine längere Einarbeitungszeit in dem Sinne gewährt wurde, dass erst nach zwei Jahren die gleiche Leistung wie bei langjähriger Mitarbeit erwartet wurde. Dieser enorme Druck auf neue Mitarbeiterinnen oder Mitarbeiter provoziert Fehler und kann insofern eine kurzsichtige und irrige Strategie sein. Die Vorgabe, Zeit einzusparen, hat auch Auswirkungen auf Situationen des sozialen Zusammenlebens wie Geburtstagsfeiern und Kaffeepausen. Hat man vor ein paar Jahren noch gemeinsam auf Geburtstage angestoßen, so ist nun die Zeit dafür an vielen Arbeitsstellen zu knapp. Der informelle soziale Austausch in der Kaffeeküche ist beim letzten Arbeitgeber von L. durch die Einführung der Regel, auch kurze Kaffeepausen „auszustempeln“, zum Erliegen gekommen. Bei ihrer neuen Tätigkeit im öffentlichen Dienst erlebt sie hingegen, dass derartige Praktiken dort heute noch gelten und meint: „Das ist wie in meiner Firma vor 20 Jahren." Dementsprechend äußerten mehrere Teilnehmende den Wunsch, nicht mehr in der Privatwirtschaft, insbesondere nicht bei einem internationalen Konzern, beschäftigt zu sein.

Während diese Veränderungen nur schwer greifbar sind und eher subjektive Eindrücke widerspiegeln, berichtet beispielsweise L. auch, wie sich die gut dokumentierte geringe Reallohnsteigerung während der letzten Jahre (Hermann/Flecker 2014: 180-181) in ihrem persönlichen Erleben niedergeschlagen hat: Ab 2007 wurde bei ihr nur mehr der Lohnanteil nach Kollektivvertrag erhöht, die Überzahlungen nicht mehr, wodurch ihre jährliche Gehaltserhöhung ab dann immer weit unter der Inflation lag. Ferner berichtet sie, dass sogenannte „All-in-Verträge“ üblich wurden, mit denen Überstunden pauschal abgegolten werden: „Wenn es jetzt 60 Stunden Arbeitszeit durch Dienstreisen waren, hat es keinen Zeitausgleich mehr gegeben. Das geht natürlich dann auf die eigene Substanz."

Eine dominierende Erzählfigur stellt jene vom „Mensch als Werkzeug“ dar. Der Mensch zähle in den Unternehmen immer weniger, er wird verdinglicht und je nach Bedarf wie ein Werkzeug ausgetauscht. Die Teilnehmenden stellen zunehmenden Konkur- 
renzdruck fest, der sich auch in der Entwicklung der Arbeitsstunden niederschlägt: Einerseits sind bei ihren früheren Arbeitgebern Beschäftigte abgebaut worden, andererseits ist Druck auf die verbliebenen Arbeitnehmerinnen und Arbeitnehmer ausgeübt worden, unbezahlte Überstunden zu leisten. Z. äußert sich dahingehend, dass ihr früherer Arbeitgeber ihrer Einschätzung nach die Wirtschaftskrise als Vorwand genutzt hat, um den Druck auf die Mitarbeitenden zu erhöhen. Mehrere Teilnehmende haben in ihren letzten Arbeitsstätten eine Art Wettbewerb unter den Mitarbeiterinnen und Mitarbeitern darum erlebt, wer am längsten arbeitet und dadurch die eigene Unverzichtbarkeit unter Beweis stellt. Dieses Hamsterrad führt zu einer noch intensivierten Polarisierung zwischen jenen, die in dieser Arbeitswelt mithalten können, und jenen, die draußen sind. Dazu meint Z.: „Ich finde es unsozial, regelmäßig 60 Stunden zu arbeiten und davon 20 Stunden Gratis-Überstunden, weil damit anderen Beschäftigung verwehrt bleibt." Aber auch das „Drinbleiben“ geht oft mit hohen Kosten für das Individuum einher. Die potenzielle Beeinträchtigung des Rechts (1) auf Gesundheit durch zu hohen arbeitsbezogenen Druck in Form von Stresssymptomen bis hin zum Burnout sind bekannt. Die gesundheitlichen Probleme der Teilnehmenden sind dafür illustrativ: Die jahrelange Beschäftigung in prekären Arbeitsverhältnissen hat bei O. zu psychosomatischen Beschwerden bis hin zum Aussetzen des Atems geführt. Ähnlich dramatisch ist das Beispiel von G., die - wie sie selbst sagt - sich oft so unter Druck gesetzt hat, dass ihr Herz angegriffen war und sie sich einer Notoperation unterziehen musste. L. litt unter Burnout. B. musste seinen Beruf in der Gastronomie wegen eines Bandscheibenvorfalls aufgeben und H. den seinen in der Industrie. Die Fähigkeit und das Recht (8), einer Beschäftigung nachzugehen, stehen damit im Widerspruch zu anderen Rechten und Fähigkeiten. Die Bedingungen der Arbeitswelt werden von den Teilnehmenden hinsichtlich vieler Facetten als schädlich für ihre Rechte und Capability beurteilt. Ihre Arbeitsverhältnisse hatten schleichend viele Aspekte eingebüßt, die sie aus guten Gründen wertgeschätzt hatten. Diese Entwicklung schreiben die Betroffenen nicht ursächlich der Finanz- und Wirtschaftskrise zu, sondern verorten sie in einem Prozess, der dadurch verstärkt und beschleunigt wurde, insbesondere auch, weil alternative Wahlmöglichkeiten plötzlich als stark eingeschränkt erlebt wurden.

„Ich hätte die ganzen Jahre kündigen können, das stimmt. Aber, wie hat es ausgesehen am Arbeitsmarkt?
Und das hat mich überhaupt in diesem ungesunden Zustand dort gehalten - die prekäre Situation am Arbeitsmarkt."(L.)

In der Steuerungsgruppe wurde von Expertinnen und Experten der Praxis aus dem Bereich berufliche Neuorientierung die These bestärkt, dass die Chancen für einen beruflichen Neuanfang für Ältere während der letzten Jahre deutlich gesunken sind.

\subsection{Kontext: Die Finanzkrise 2008 und ihre Folgen für Österreich}

Österreich ist im Vergleich $\mathrm{zu}$ anderen Staaten der EU gut durch die Krise gekommen. Zuvor hatte es - aufgrund der Lage im Herzen von Europa und der starken Exportorientierung - aus volkswirtschaftlicher Sicht stark von der Einführung des Euro sowie der EU-Osterweiterung profitiert. Makroökonomisch wird das, was die Betroffenen als „Hamsterrad“ bezeichnen, als mehrere Jahre andauernde Phase beschrieben, die von vergleichsweise hohem Wirtschaftswachstum, sinkender Erwerbsarbeitslosigkeit sowie ab 2006 erstmals wieder signifikanten Reallohngewinnen für Arbeitnehmerinnen und Arbeitnehmer gekennzeichnet war (Hermann/Flecker 2014). Diese Phase wurde durch die Finanz- und Wirtschaftskrise ab 2008 beendet, wodurch sich das „Hamsterrad“ für die damals noch beschäftigten Teilnehmenden noch schneller zu drehen begann.

Vom Einsetzen der Wirtschaftskrise ab 2008 waren dennoch zwei Sektoren in Österreich besonders betroffen: zum einen die Banken und zum anderen die österreichische Exportwirtschaft, insbesondere die Produktionsbetriebe. So brachen die Exporte im Jahr 2009 um über $20 \%$ ein. Nachdem die österreichische Wirtschaft 2008 noch 2 \% Wachstum verzeichnen konnte, geriet sie 2009 in die tiefste Rezession seit 1945, das Bruttonationalprodukt schrumpfte um 3,6 \% (Hermann/Flecker 2014).

Die unmittelbare Krisenpolitik der österreichischen Regierung konzentrierte sich auf Stabilisierungsprogramme für die besonders betroffenen Sektoren und Konjunkturpakete. Diese beinhalteten unter anderem Steuererleichterungen, eine Verschrottungsprämie für Altautos bei Kauf eines Neuwagens („Ökoprämie“), öffentliche Investitionen in Infrastruktur und staatlich geförderte Kredite für Kleine und Mittlere Unternehmen („Mittelstandsmilliarde“). Nach einem heftigen, aber kurzen Einbruch hatten sich die Exporte schon Ende 2009 wieder fast vollständig erholt. Dem Ban- 
Abbildung 4: Entwicklung der Arbeitslosigkeit und des Anteils älterer Arbeitsloser 2007-2016

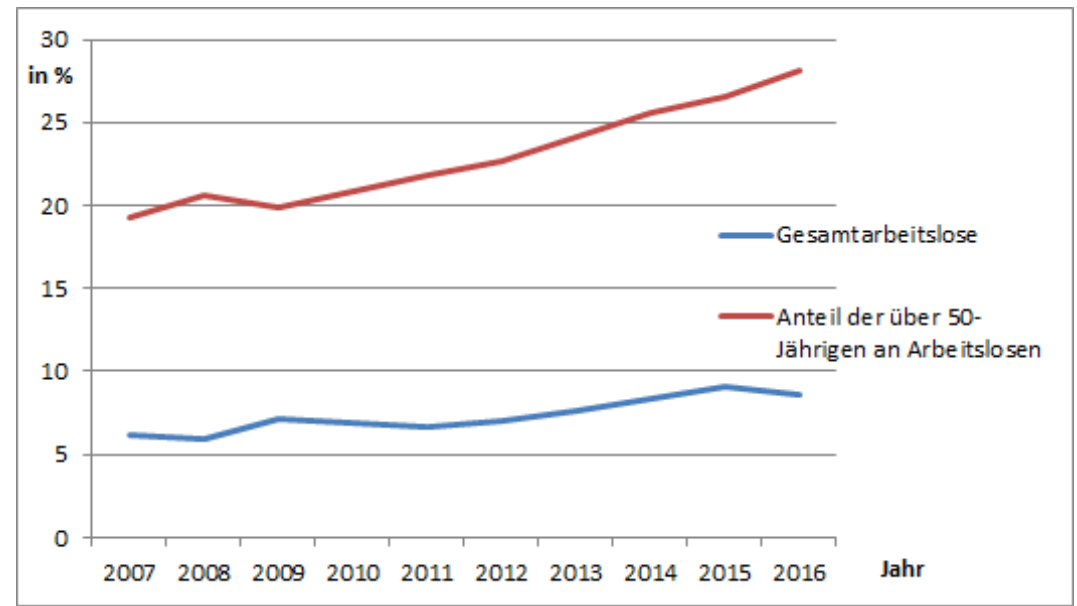

Quelle: eigene Darstellung auf der Grundlage von Daten des Arbeitsmarktservice (AMS)

kensektor wurde mit einem Rettungspaket in Höhe von 100 Milliarden Euro unter die Arme gegriffen (daraus entstanden der öffentlichen Hand bis Ende 2016 je nach Berechnungsmethode zwischen 9 und 14 Milliarden Euro tatsächlich defizitwirksame Ausgaben, vgl. orf.at 2016). Als Folge der staatlichen Ausgabenerhöhungen zur Bekämpfung der Krise erhöhte sich das jährliche Budgetdefizit in den Jahren 2009 (auf 5,3 \% vom BIP) und 2010 (4,4 \%) stark (2008: 1,4 \%) (Statistik Austria 2015). Auch das Land Salzburg hatte, wie andere österreichische Bundesländer, Städte und Gemeinden, hochriskante Finanzgeschäfte getätigt, die im Zuge der Finanzkrise zu hohen Spekulationsverlusten führten, wie Ende des Jahres 2012 ans Tageslicht kam. In Kombination mit durch die Krise gesunkenen Einnahmen und gestiegenen Ausgaben war die Neuverschuldung im Jahr 2013 deutlich höher als budgetiert. Im Budget 2014 wurden umfassende Sparmaßnahmen vorgenommen, die Landesregierung betonte die labile Situation des öffentlichen Haushalts und kündigte auch für die Folgejahre Sparbudgets an (orf.at 2013).

Um Kündigungen $\mathrm{zu}$ vermeiden, wurden auf Bundesebene zwei Arbeitsmarktpakete aufgelegt, die primär darauf abzielten, als Alternative zu Stellenabbau die interne Flexibilität von Unternehmen zu erhöhen, indem die Arbeitsstunden pro Arbeitnehmendem und die Produktivität pro Stunde verringert werden. Die wichtigste Reform war der vereinfachte und flexiblere Zugang für Unternehmen zu Kurzarbeit. Diese wurde auf Basis einer Vereinbarung der österreichischen Sozialpartner umgesetzt und gilt als zentraler Faktor für den vergleichsweise moderaten Anstieg der Erwerbsarbeitslosigkeit um 1,4 \%. Im EU-Vergleich blieb die Arbeitslosigkeit in Österreich weiterhin niedrig. Die stabilisierenden Effekte der ergriffenen Maßnahmen kamen jedoch, entsprechend der Aufsplitterung des österreichischen Arbeitsmarkts in gut geschützte Kern- und flexibilisierte Randbelegschaften, fast ausschließlich qualifizierten Arbeitnehmerinnen und Arbeitnehmern mit unbefristeten Verträgen zugute. Demgegenüber waren von der steigenden Erwerbsarbeitslosigkeit anfangs vor allem Beschäftigte in der Leiharbeitsbranche betroffen, deren Anzahl in den Jahren vor der Krise stark zugenommen hatte. In der Rezession waren sie die ersten, die freigestellt wurden. Im Industriesektor betrug der Rückgang 32,8 \% der dort beschäftigten Leiharbeitskräfte zwischen Juli 2008 und 2009 (Basso et al. 2011: 11; Hermann/Flecker 2014). Insgesamt waren von diesem ersten Anstieg der Erwerbsarbeitslosigkeit jüngere Arbeiter und Arbeiterinnen, Migrantinnen und Migranten sowie niedrig Qualifizierte besonders betroffen. Verhältnismäßig wurden auch mehr Männer als Frauen arbeitslos (Hermann/Flecker 2014).

Von der zweiten Anstiegswelle bei der Erwerbsarbeitslosigkeit ab 2012 sind, neben Menschen mit Migrationshintergrund und Niedrigqualifizierten, Personen über 50 bzw. sogar schon ab 45 Jahren besonders stark betroffen (Abb. 4). Das liegt zum einen daran, dass diese Gruppe durch den demografischen Wandel zahlenmäßig größer wird, und zum anderen steht sie dem Arbeitsmarkt durch Erhöhung des tatsächlichen Pensionsantrittsalters länger zur Verfügung. Das im Vergleich zum EU-Durchschnitt niedrige faktische Pensionsantrittsalter (Abschnitt 3.1) wirkt zwar abschwächend auf die Arbeitslosenzahlen, deutet aber auch darauf hin, dass die österreichische Wirtschaft noch nicht darauf eingestellt ist, die größer werdende 
Gruppe älterer Arbeitnehmerinnen und Arbeitnehmer bis zum Pensionsantritt zu beschäftigen.

Im Land Salzburg stieg zwischen 2010 und 2014 die Zahl der Erwerbsarbeitslosen um 27,8 \% und in der Stadt Salzburg sogar um 42,7 \%. Aufgrund geringer Versicherungsleistungen erhielt ein Viertel der arbeitslos gemeldeten Personen zusätzliche Leistungen aus dem Sozialbudget des Landes Salzburg (Bedarfsorientierte Mindestsicherung), um ihre Existenz zu sichern. Die Zahl der Mindestsicherungsbeziehenden hat sich zwischen 2013 und 2014 um rund 8 \% erhöht. 70 \% der Anspruchsberechtigten hatten zwar ein Einkommen, konnten jedoch damit aufgrund steigender Kosten ihren Lebensunterhalt nicht vollständig bestreiten (Land Salzburg 2015).

Während die Erwerbssituation als Folge dieser Entwicklungen für viele Menschen schwieriger wurde, erfolgten im Unterschied zu anderen EU-Staaten keine weitreichenden, sondern nur punktuelle Kürzungen im Sozialbereich oder bei öffentlichen Dienstleistungen (Fink 2015a). Die stabilisierende Funktion sozialstaatlicher Leistungen bewirkte, dass der Anteil armutsgefährdeter Personen sich durch die Krise in Österreich nicht erhöht hat (BMASK 2014: 385).

\subsection{Ergebnisse: (Soziales) Leben im freien Fall}

„... die erste Frage ist nicht: Wie geht es dir so? Sondern: Was machst du jetzt?" (K.)

Selbst wenn sie die Krise nicht als unmittelbare Ursache ihrer Arbeitslosigkeit erlebt haben, gehören die Teilnehmenden der Studie zu jener Gruppe, die davon besonders betroffen ist, wie Abbildung 4 zeigt. Die Auswirkungen der Arbeitslosigkeit auf das Recht (2) auf angemessenen materiellen Lebensstandard der Betroffenen sind sehr unterschiedlich, abhängig von der vorher bestehenden Ressourcenausstattung (z. B. Eigentumswohnung vs. Mietwohnung, Ersparnisse, Höhe des Arbeitslosengeldes) und Familienkonstellation (z. B. berufstätige vs. pflegebedürftige Familienangehörige). Die Krise hat diesbezüglich aufgrund ausbleibender Kürzungen der Sozialleistungen keine wesentliche Veränderung mit sich gebracht. Unabhängig davon macht es allen Mitgliedern der Betroffenenforschungsgruppe zu schaffen, dass die Frage nach der Berufstätigkeit derart im Mittelpunkt des Denkens im sozialen Umfeld steht. Wer keine Arbeit hat, fällt aus vielen sozialen Netzen heraus und ist in Bezug auf das Recht (5) auf Familien- und gesellschaftliches Leben beeinträchtigt. Erwerbsarbeitslosigkeit ist erklärungs- bedürftig, wird primär als individuelle Problemlage wahrgenommen und für das Gegenüber ist es unangenehm, von der schwierigen Lage zu hören, wie K. beobachtet hat:

„(...) und dann musst du aufpassen auch noch, weil $d u$ schockierst sie ja, wenn du ihnen die Wahrheit sagst, dass du eigentlich komplett am Boden bist, knapp vor unter der Brücke, dann schockierst du sie."

Es war für K. daher eine große Überwindung, eine Sommersaison dort als Servicekraft zu arbeiten, wo ihre ehemaligen Nachbarinnen und Nachbarn des schönen Salzburger Luxus-Stadtviertels ihre Freizeit verbrachten: „Das war eine Mutprobe für mich." Insgesamt stellt sie fest: „Mein komplettes soziales Umfeld, meine Familie ausgenommen, hat sich komplett ausgetauscht. Aus meinem früheren Freundeskreis gibt es niemanden mehr."

Nicht nur bei ihr, sondern bei den meisten Betroffenen sind die familiären Beziehungen stabiler als Freundschaften. Intakte Partnerschaften können sowohl die materiellen Folgen abmildern als auch eine wichtige Stütze bei der Jobsuche sein, wie es bei L. und H. der Fall ist. Wenn Trennung und Scheidung schon länger zurückliegen (wie bei Z. und M.) oder wie bei B. - die Partnerin verstorben ist, gibt es von dieser Seite zwar keine Unterstützung, dafür stellt aber die Erwerbsarbeitslosigkeit dann auch keine Belastung für die Partnerschaft dar, wie es bei G. der Fall war. Schließlich kann aber das Auseinanderbrechen der Partnerschaft auch die Ursache der Arbeitslosigkeit sein, wie im Fall von K., die in der Consulting-Firma ihres Mannes gearbeitet hatte, welche im Zuge der Finanzkrise insolvent wurde. Ihr Leben fühlte sich tatsächlich an wie ein „freier Fall“:

„Bei uns ist praktisch wirklich alles auseinandergefal-

len. Die Firma weg, das Haus weg, die Existenz weg, der Sohn hat mit der Schule aufgehört und ich habe bei null wieder angefangen."

Es ist für die Teilnehmenden besonders belastend, dass sie ihre Kinder nicht mehr im selben Maße wie früher unterstützen können. Sie möchten sie in deren Recht auf (3) Bildung und Lernen unterstützen, um ihnen für ihren weiteren Lebensweg gute Chancen zu eröffnen. Wie das Zitat von K. zeigt, konnte sie das Schulgeld für die Privatschule nicht mehr zahlen und musste erleben, wie ihr Sohn durch die berufliche und private Krise seiner Eltern seine Bildungsmotivation verloren hat und frühzeitig von der Schule gegangen ist, um Geld zu verdienen. Bei L. hat ihre Arbeitslosigkeit aufgrund von Ersparnissen zwar nicht derart dramati- 
sche Folgen gehabt, doch die Aussicht, die finanzielle Unterstützung für ihre Tochter bei länger andauernder Arbeitslosigkeit kürzen zu müssen, beschäftigte sie stark. O. hat ihre Tochter allein erzogen und war die ganze Zeit über in einer prekären Situation (keine Unterhaltszahlungen, drei Jobs nebeneinander, Selbstständigkeit als Pädagogin etc.). Sie ist einerseits froh und stolz, ihrer Tochter zurzeit ein Studium ermöglichen zu können, muss dafür aber andererseits einmal mehr alles Geld zusammenkratzen. M. berichtet davon, dass seine Tochter sich selbst - erfolgreich - bei einer Privatschule beworben hat und dafür ein Stipendium bekommt. Es ist ihm anzumerken, wie sich der Stolz über die Tochter mit dem Bedauern mischt, nicht mehr beitragen zu können.

Hinsichtlich der Beziehung zu den Eltern gibt es beides: Sie gewähren ihren Kindern teils Unterstützung - in Form von Verständnis, aber auch in Form von materieller Hilfe -, aber sie benötigen auch selbst Unterstützung in Form von Pflege. Zwei Teilnehmerinnen pflegen ihre Eltern. Beide haben eine innige, wenn auch nicht konfliktfreie Beziehung zu ihren Eltern und erleben die Pflege gleichermaßen als lohnend wie auch stark belastend. Während K.s Eltern pflegebedürftig wurden, als K. bereits arbeitssuchend war, hat F. ihre Selbstständigkeit schließlich aufgeben müssen, um ihre Eltern pflegen zu können. Zwar hat die Politik mit der Einführung der Pflege- und Hospizkarenz zum 1. Januar 2014 auf derartige Probleme reagiert, aber das hat keine schlagartige Veränderung der Einstellung gegenüber der Pflege bewirkt: Die Betroffenen berichten, dass die Pflege kaum Wertschätzung genießt und primär als private Verantwortung der (in der Regel weiblichen) Hauptpflegeperson gesehen wird. Auf die eingangs erwähnte Frage: „Was machst du?" wird die Antwort: „Ich pflege meine Eltern.“ nicht als ausreichend empfunden. Pflege wird nicht als tagesfüllende Tätigkeit angesehen, die wie eine Berufstätigkeit einen Beitrag zur Gesellschaft leistet. Beide Teilnehmerinnen erzählen eindrücklich, wie wenig Unterstützung sie in der Pflege erfahren: Die Beantragung von Pflegekarenz ist zwar möglich, aber weder bei Arbeitgebern akzeptiert noch beim Arbeitsmarktservice (im Folgenden AMS) gut bekannt. Wie lange die Pflege dauert, ist nicht absehbar. Pause bzw. Urlaub von dieser psychisch äußerst fordernden Tätigkeit ist jedoch nur dann gut möglich, wenn ein Ersatz für den entsprechenden Zeitraum gefunden wird. Dies ist nicht nur schwierig, weil kaum jemand die Pflege übernehmen mag, sondern auch weil die Pflegenden selbst ein schlechtes Gewissen plagt, die Sorgearbeit - auch nur für kurze Zeit - abzugeben.

Z.s Mutter ist bereits vor einigen Jahren nach langer Pflegebedürftigkeit verstorben. Das Erbe hat mit dazu beigetragen, dass Z. sich eine Eigentumswohnung kaufen konnte und nun diesbezüglich abgesichert ist. Dies ist ein großes Glück, wie L. bestätigt:

„Außer man hat Rücklagen, hat geerbt oder so, o. $k$. Aber Rücklagen bilden ist ja auch immer schwerer geworden. Also, wenn ich jetzt sehe, das Leben meiner Eltern vergleiche und meines, die haben sich ja wirklich aus dem Nichts eine Eigentumswohnung kaufen können. Das kann meine Generation nicht."

L. zog, noch bevor sie arbeitslos wurde, ihr Gebot für eine Eigentumswohnung wieder zurück, weil die finanzielle Belastung zu groß gewesen wäre. Sie hat das Glück, schon lange in ihrer Wohnung zu sein und daher eine relativ niedrige Miete zu zahlen. Der hohe Anstieg der Immobilienpreise in Salzburg hat auch zum Anstieg der Mieten geführt, wie einige ProjektTeilnehmende erfahren haben. So musste sich K. mit zunehmender Dauer der Arbeitslosigkeit wiederholt eine günstigere Wohnung suchen. O. ging eine Betreuungsverpflichtung für eine ältere Dame als Gegenleistung für eine kostengünstige Wohnmöglichkeit ein.

Insgesamt zeigt besonders der Fall von K., dass oftmals zur Arbeitssuche noch weitere Probleme kommen, für die jeweils unterschiedliche Stellen zuständig sind: K. musste die Unterhaltszahlungen ihres geschiedenen Mannes einfordern, Unterstützung für das Schulgeld ihres Sohnes beantragen, sich - wiederholt - preiswertere (und kleinere) Wohnungen suchen, Mindestsicherung für sich und Pflegegeld für ihre Eltern beantragen. Sie ist dabei von keiner Stelle beraten worden, in welcher Reihenfolge dies am besten zu geschehen hat und was es dabei zu beachten gilt, wie beispielsweise Fristen. Sie fühlte sich auf sich allein gestellt und vermutet, dass die jeweils zuständigen Personen in den Behörden ebenso wie sie selbst von ihrer Situation überfordert waren, da so viele „Baustellen“ (Existenzsicherung, Arbeit, Gesundheit, Familie) zeitgleich zu bearbeiten waren.

„Man schickt dich im Kreis, keiner ist für dich zuständig, du musst dir deinen Weg schlussendlich wirklich selbst suchen und dranbleiben bei allen Instanzen und du musst dir das Szenario, das für dich persönlich am besten passt, auch selbst zuschneiden."

Dies entspricht der Feststellung in der Fachliteratur, dass die individuelle Betreuung eines der großen Probleme im österreichischen Sozialstaat ist (Fink 2015b: 
6). Dies hängt u. a. damit zusammen, dass kumulierten Problemlagen in krisenhaften Lebenssituationen in Österreich ein ebenso komplexes Unterstützungssystem gegenübersteht (siehe auch: Egger-Subotitsch, 2014: 47 zum Thema psychisch belastete Arbeitslose im Burgenland). Als positives Beispiel für individuelle Beratung verweisen die Teilnehmenden auf das Case-Management bei der PVA (Pensionsversicherungsanstalt), in dessen Rahmen ein individueller Versorgungsplan erstellt wird (Arbeiterkammer Wien 2014).

Menschen, die längere Zeit unfreiwillig ohne Erwerbsarbeit sind, haben oft mit vielen Problemen zu kämpfen, wie unsere Forschungsergebnisse verdeutlichen: mit gesundheitlichen und psychischen, die teils erst zur Arbeitslosigkeit geführt haben (Abschnitt 3.2) und teils dadurch verstärkt werden; mit der deutlichen Verknappung des Budgets, die zu Einschnitten beim Wohnen, aber auch bei den Unterstützungsmöglichkeiten anderen gegenüber führt; sowie mit den ganz normalen Herausforderungen wie der Pflegebedürftigkeit der Eltern. Vor diesem Hintergrund trifft es die Projekt-Teilnehmenden stark, dass in der Gesellschaft das Bild der „sozialen Hängematte“ wieder häufiger bemüht wird. So wird ihrer Wahrnehmung nach suggeriert, die Erwerbsarbeitslosen ließen es sich auf Kosten der Gemeinschaft gut gehen.

Das Bild des „freien Falls" beziehen sie nicht nur auf ihre sozialen Beziehungen im engeren Sinn, sondern auf ihr Leben als Ganzes, das sie als sozial eingebettet und (trotzdem und deswegen) als sehr fragil wahrnehmen, sobald die zentrale Verortung über die Erwerbstätigkeit wegfällt. L. drückt dies in einem abschließenden Statement zu ihrer Biografie folgendermaßen aus:

„Die Erfahrung ist für mich, dass du auf so dünnem Eis stehst. Solange du einen Job hast, hast du ja alles. Es ist alles prima. Aber sobald das wegfällt, brichst du ein. Das ist einfach so schrecklich."

\subsection{Ergebnisse: Blackbox Arbeitsmarktservice}

„Die haben eine Spannbreite, wie sie dich behandeln. Wenn du brav bist, lassen sie dich in Ruhe, wenn nicht, drangsalieren sie dich." (H.)

Die Interaktion mit staatlichen Institutionen nimmt eine große Rolle in den erzählten Biografien der Teilnehmenden ein. Viele berichten von unangenehmen und energiezehrenden Erlebnissen. L. hat nach ihrem Burnout, aufgrund dessen sie gekündigt wurde, den Kontakt zu öffentlichen Institutionen wie dem AMS und der Krankenversicherungsanstalt so weit wie möglich vermieden, da sie eher negative Erfahrungen gemacht hatte:

„Sie [eine Wahlärztin] hat mich ein bisschen geschützt vor der Gebietskrankenkassa. Ich meine, zu dem Termin immer hinzugehen, ist mir nicht erspart geblieben. Da gibt es sehr verständnisvolle [Ärztinnen und Ärzte], die sehr nett mit einem reden und auch beraten, und dann gibt es welche (...) bei einer, da bin ich zwei Mal in Tränen ausgebrochen."

K. zieht folgendes Resümee: „(...) du kommst auf keinen Fall gestärkt aus solchen Institutionen heraus, das ist meine Erfahrung. Vielleicht ist es anderen besser ergangen, aber mir hat das jedes Mal (Lachen) eigentlich noch einen Tritt ins Knie gegeben."

Die wichtigste zuständige Stelle für Arbeitssuchende ist jedoch das Arbeitsmarktservice in Österreich. Die Projekt-Teilnehmenden haben während ihrer langjährigen Interaktion mit dem AMS sehr durchwachsene Erfahrungen gemacht. Einige sind gut beraten worden und haben AMS-Beraterinnen und -Berater erlebt, die engagiert sind und sich bemühen, ihre Klientel zu unterstützen. Sie erzählen von kompetenten Beratern und Beraterinnen, die verbindliche Auskunft geben können, und auch von solchen, die selbst deprimiert bzw. resigniert sind, weil sie besonders während der vergangenen Jahre nicht mehr für die Arbeitssuchenden tun können: „Ich habe weder Jobs für Jüngere noch für Ältere." Sie erzählen jedoch auch von häufigen Wechseln in der Betreuung, die es nötig machen, den eigenen Leidensweg ein ums andere Mal zu erzählen, während man gleichzeitig das Gefühl hat, dass einem nicht richtig zugehört wird. Thematisiert wurde auch, dass das AMS von seiner Grundstruktur (z. B. standardisierte Zuteilung nach Geburtsmonat statt inhaltlicher Kriterien, enges Zeitkorsett für Beratungsgespräche) nicht dafür gerüstet ist, Personen mit multidimensionalen Problemlagen gut zu beraten. K. hat diesbezüglich die Erfahrung gemacht, dass ihre psychischen Vermittlungshindernisse nicht konstruktiv berücksichtigt, sondern mit dem simplen Vorschlag, eben nicht Vollzeit zu arbeiten, abgetan wurden:

„Die sind ja da unsensibel (...). Ich hab von meiner Ärztin weiß Gott was für Atteste bringen können, da ist drauf gestanden, sie leidet seit Jahren an einem depressiven Belastungssyndrom der feinsten Sorte, das ist denen beim AMS wurscht."

G. dagegen wurde nach ihrem Burnout einer speziellen Abteilung beim AMS zugeteilt, deren Mitarbei- 
terinnen und Mitarbeiter sie sehr einfühlsam beraten haben und keinen Druck ausübten, was für ihre Regeneration sehr hilfreich war.

Problematisiert wurde in der Gruppe auch die häufige Erfahrung, dass sie unerfahrenen Mitarbeiterinnen oder Mitarbeitern des AMS gegenübersitzen, die offenbar viele Programme und Regelungen nicht kennen und denen sie - die Arbeitssuchenden - diese Regelungen und ihre damit verbundenen Rechte erst erläutern müssen bspw. den Bemessungsgrundlagenschutz $^{10}$ für das Arbeitslosengeld (Arbeitsmarktservice Österreich 2015C: 58) oder die Pflegekarenz. ${ }^{11}$ Ein illustratives Beispiel ist der Bemessungsgrundlagenschutz: Über dessen Bestehen herrschte bspw. in der Gruppe große Unklarheit, obwohl alle aufgrund ihres Alters schon darunter fallen müssten. Eine Teilnehmerin erzählte, dass sie sich nicht getraut habe, einen befristeten Job mit niedrigerem Gehalt anzunehmen, aus Angst durch das danach verringerte Arbeitslosengeld in Armut abzurutschen. Das Wissen um den Bemessungsgrundlagenschutz hätte es ihr erlaubt, diese Arbeit anzunehmen.

Die Teilnehmenden haben auch vielfach die Erfahrung gemacht, dass sie über Programme nur auf spezielle Nachfrage ihrerseits informiert wurden und, wenn überhaupt, nur durch große Hartnäckigkeit zur Teilnahme zugelassen wurden. Inwiefern dies eine gezielte Politik des AMS oder tatsächlich individuelles Nichtwissen der Grund ist, wurde kontrovers diskutiert. Deutlich wurde jedoch die Beeinträchtigung des Rechts (7) auf Rechtssicherheit durch mangelnde Information und Transparenz oder gar Fehlinformation. Die Qualitätssicherung durch standardisierte Kundenzufriedenheitsbögen, wie es sie auch beim AMS gibt, hilft diesbezüglich nicht wirklich weiter, denn es fehlt die Möglichkeit, Kommentare einzufügen oder gezielte und gleichzeitig geschützte Rückmeldungen zu geben.

10 Der Bemessungsgrundlagenschutz besagt, dass zur Ermittlung der Höhe des Arbeitslosengeldes bei Arbeitssuchenden, die über 45 Jahre alt sind, das Entgelt herangezogen wird, das bereits vorher als Grundlage zur Berechnung diente. Arbeitssuchende in diesem Alter verlieren also nicht den Anspruch auf ein höheres Arbeitslosengeld, wenn sie eine schlechter bezahlte Stelle annehmen als sie sie zuletzt innehatten.

11 Wer vom AMS Leistungen bezieht, kann sich davon für die Pflege eines Angehörigen ab der Pflegestufe 3 für bis zu drei Monate abmelden, um die Absicherung über die Kranken- und Pensionsversicherung in Anspruch zu nehmen, siehe http://www.ams.at/service-arbeitsuchende/ finanzielles/leistungen/familienhospiz-pflegekarenz.
Diesen Erfahrungen entspricht, dass im Jahr 2014 bei der Volksanwaltschaft, die in Österreich verfassungsmäßig für die Kontrolle der öffentlichen Verwaltung sowie den Schutz und die Förderung der Menschenrechte zuständig ist, um $70 \%$ mehr das AMS betreffende Beschwerden eingingen als im Jahr 2015. Diese betrafen Fragen nach der Rechtmäßigkeit von Zuweisungen zu (Kurz-)kursen, die Qualität von Maßnahmen sowie die Kompetenz der AMS-Berater (derStandard.at 2015). Dieser Anstieg ereignete sich parallel zur steigenden Zahl an Arbeitssuchenden und kann als indirekte Krisenfolge betrachtet werden, weil die pro Kopf verfügbaren Mittel und die pro Kunde oder Kundin zur Verfügung stehende Beratungszeit dadurch abnahmen. Aufgrund eines Strategiewechsels des AMS reduzierte sich die Zahl der Schulungsteilnehmenden im Jahr 2015 dann deutlich, da weniger, aber dafür längere Kurse angeboten und unbeliebte Standardkurse reduziert wurden (orf.at 2015). Fragen der Servicequalität und Rechtssicherheit werden jedoch von den Teilnehmenden weiterhin als sehr relevant eingestuft.

Problematisiert wurde auch die grundsätzliche Unausgewogenheit, dass die Teilnehmenden am AMS eher als Bittsteller denn als Anspruchsberechtigte behandelt werden, obwohl es sich bei Arbeitslosengeld und Arbeitsvermittlung eigentlich um durch einen Rechtsanspruch gedeckte Leistungen handelt. Dies erscheint ihnen besonders paradox, da es in der Wahrnehmung der Teilnehmenden zum Zeitpunkt der Erhebung schlicht keinen ausreichenden Zugang zu existenzsichernden und befriedigenden Arbeitsplätzen gibt, weil die Nachfrage am Erwerbsarbeitsmarkt deutlich größer als das Angebot ist. Vollbeschäftigung scheint demnach in absehbarer Zukunft nicht wieder erreichbar. Gleichzeitig machen ihrer Einschätzung nach dennoch „alle so weiter wie bisher“. Dadurch werde der Druck unverhältnismäßig stark den arbeitslosen Individuen zugeschoben, obwohl es sich im Kern um ein strukturelles Problem handelt. Die Arbeitsmarktpolitik wird deshalb als inadäquat erlebt. Die Erwartung, dass das AMS tatsächlich bei der Arbeitssuche als Vermittlerin hilfreich ist, haben die Teilnehmenden größtenteils nicht (mehr). Um jedoch nicht drangsaliert zu werden, müsse man sich oft verstellen, beispielsweise wenn man die Sinnhaftigkeit einer Vorgabe nicht sieht oder sich nicht in der Lage fühlt bzw. willens ist, die vom AMS eingeforderten Pflichten $\mathrm{zu}$ erfüllen. Die Interaktion mit dem AMS wird insofern als „sorgfältig geplante Frustration“ (Sedmak et al 2014: 
35) erlebt, die das Vertrauen in die staatlichen Institutionen und die eigenen Fähigkeiten schwächt.

Was die Arbeitsbedingungen bei angebotenen Stellen betrifft, berichten mehrere Teilnehmende, dass es kaum Jobangebote über Vermittlung des AMS gebe und wenn, dann seien diese oft völlig unpassend für die Qualifikation. Die Qualität dieser Jobangebote wird von mehreren Teilnehmenden als schlecht empfunden, da viele Angebote Leiharbeit, geringe Bezahlung oder andere Aspekte prekärer Arbeit beinhalten. Besonders kritisch gesehen wird von M. die Vermittlung an Zeitarbeitsfirmen durch das AMS, da diese Arbeitsplätze anbieten, durch die man von wesentlichen Arbeitnehmerrechten ausgeschlossen bleibt. Als Leiterin von Callcentern hat L. erlebt, wie das Risiko während der vergangenen Jahre von Seiten der Unternehmen auf die Arbeitnehmerinnen und Arbeitnehmer abgewälzt wurde, indem die Arbeit aus dem Konzern ausgelagert und den Mitarbeiterinnen keine festen Verträge angeboten, sondern sie als „freie Dienstnehmerinnen“ eingestellt wurden. Diese Verträge sehen zwar Leistungen der sozialen Sicherung (Pensions-, Kranken-, Unfall- und Arbeitslosenversicherung) vor, jedoch kommen das Arbeitsrecht und Kollektivverträge bzw. Mindestlohntarife für sie nicht zur Anwendung. (WKO o. J.) Die vereinbarten Honorare sind deshalb der geforderten Leistung oft nicht angemessen. Insofern ist dies eine Form der Prekarisierung (Kraemer 2009).

Der Druck, auch qualitativ als schlecht oder sogar schädlich empfundene Arbeit anzunehmen, steigt mit der Dauer der Arbeitslosigkeit, da Berufs- und Entgeltschutz zeitlich befristet gelten. Das Risiko einer Kürzung oder temporären Sperre der Auszahlung von Arbeitslosengeld, Notstandshilfe und Mindestsicherung bei Handlungen, die vom AMS als „Arbeitsvereitelung " betrachtet werden könnten, ist für die Teilnehmenden durchaus präsent. Das neben der Arbeitsvermittlung auch für eventuelle Sperren zuständige AMS wird deshalb von vielen als eher bedrohlich und wenig hilfreich erlebt.

Das Fehlen von substanziellen Mitspracherechten bei der Interaktion mit dem AMS wird ebenfalls problematisiert und betrifft das Recht (6) auf Teilhabe und Mitsprache. So spreche man am AMS beim sogenannten Betreuungsplan von einer „Vereinbarung“, selbst wenn in Wirklichkeit häufig die AMS-Berater bzw. -Beraterinnen die Maßnahmen einseitig festlegen und den Arbeitssuchenden sagen, sie müssen diesen unterschreiben, um Arbeitslosengeld zu bekommen. Informationen über die konkreten Rechte und Pflichten von
Arbeitssuchenden gegenüber dem AMS werden laut Erfahrung der Teilnehmenden des Öfteren nicht den gesetzlichen Grundlagen entsprechend kommuniziert. Auch der Begründungspflicht hinsichtlich der Zweckmäßigkeit von zugeteilten Maßnahmen bzw. der Ablehnung von Wünschen der Kundinnen und Kunden wird oft nur nachgekommen, wenn das von den Betroffenen energisch eingefordert wird. Insgesamt ist die Gruppe gegenüber der aktivierenden Arbeitsmarktpolitik „um jeden Preis" kritisch eingestellt, da die arbeitsmarktbezogenen Verwirklichungschancen der Betroffenen aufgrund fehlender öffentlicher Investitionen nicht gestärkt werden, während gleichzeitig Flexibilitätsanforderungen (z. B. hinsichtlich Zumutbarkeitsbestimmungen) einseitig zu Lasten der Arbeitnehmenden gestellt werden.

Als Grundtenor ist erkennbar, dass es stark von den Individuen abhängt, die aufeinandertreffen - von der Persönlichkeit, Erfahrung und dem Engagement der AMS-Mitarbeitenden wie auch vom Wissen und den Fähigkeiten der Arbeitssuchenden zu verhandeln, eigene Ziele festzulegen und sich durchzusetzen bzw. alternativ das AMS durch „Scheinhandlungen“ in Schach zu halten. Aus diesem Grund wurde die Bezeichnung „Blackbox“ für diese Institution gewählt, da die Betroffenen nie sicher sein können, womit sie $\mathrm{zu}$ rechnen haben. Insbesondere in Zeiten multipler persönlicher Krisen können Betroffene die notwendigen Ressourcen und Fähigkeiten für eine konstruktive Interaktion nicht aufbringen. L. berichtet von den Erfahrungen in ihrer Burnout-Selbsthilfegruppe, die sie veranlassten, den Kontakt so lange wie möglich hinauszuschieben: „Patienten, die wurden halt arbeitslos, waren beim AMS und das AMS hat ihnen den Rest verpasst.“

\subsection{Ergebnisse: Leben im Standby-Modus}

"Leben im Standby-Modus" wurde von Z. als passende Metapher für den Alltag in einer Phase (längerer) Erwerbsarbeitslosigkeit geprägt. Dieser Modus zeichnet sich durch Passivität und gleichzeitig hohen Energieverbrauch aus, welcher umso höher wird, je länger die Erwerbsarbeitslosigkeit andauert. Man ist in Warteposition, aber das dafür ganztägig und ohne Wochenende und Urlaub. Erwerbsarbeitssuchend zu sein, ist deshalb sehr energieintensiv, auch, weil man keine gesellschaftlich legitimierten Phasen der Regeneration zugestanden bekommt, wie es bspw. für Erwerbstätige der Urlaubsanspruch und für Seniorin- 
nen und Senioren die Pension ist. Im Gegensatz zur Regelung in Deutschland gibt es in Österreich keinen Fortbezug von Arbeitslosengeld, wenn man Urlaub im Ausland macht oder sich aus anderen Gründen außer Landes aufhält. Rein rechtlich müsste man sich immer abmelden, wenn man das Land verlässt und würde für diese Zeit keine Bezüge bekommen. In der Grenzregion Salzburg, in der es normal ist, nach Deutschland zum Einkaufen zu fahren oder dort lebende Familienmitglieder oder Freundinnen und Freunde zu besuchen, nehmen die Teilnehmenden diese formale Regelung als unverhältnismäßige Einschränkung ihrer Rechte auf (4) Bewegungsfreiheit und (5) Familien- und Privatleben wahr.

Jederzeit dem Arbeitsmarkt zur Verfügung stehen zu müssen, bedeutet auch, dass Weiterbildungen (Recht (3) auf Bildung) gegebenenfalls abgebrochen werden müssen, wenn ein potenzieller neuer Arbeitgeber darauf keine Rücksicht nimmt. Ähnliches gilt für das Familienleben und die Freizeitgestaltung. Gegebenenfalls muss man also alle Aktivitäten, die man während der Arbeitslosigkeit begonnen hat, wieder aufgeben. Diese (oft mühsam erlernten bzw. aufgebauten) Aktivitäten sind jedoch eine wichtige Bewältigungsstrategie für den Umgang mit der Arbeitslosigkeit. Sie strukturieren den Tag und erhalten so ein wichtiges Element der Arbeitsfähigkeit (Jahoda/Lazarsfeld/Zeisel 2014; Bednarek-Gilland 2015). Schnell kommt jedoch die Rede von der "Arbeitsvereitelung“ auf, wenn die Arbeitssuchenden nicht bereit (bzw. gesundheitlich in der Lage) sind, Vollzeit zu arbeiten, oder wenn sie wegen einer Weiterbildung (für die eventuell Kosten in Kauf genommen wurden) einen potenziellen neuen Job nicht schon am nächsten Tag antreten können. Der Planungshorizont wird deshalb durch die Erwerbsarbeitslosigkeit deutlich enger, die Fähigkeit in sich selbst zu investieren, nimmt tendenziell ab.

Die Einschränkung von Autonomie, Entscheidungsfreiheit und Rechten kratzt am Selbstwertgefühl der Betroffenen. Z. erklärte diesbezüglich, wie frustrierend es für sie sei, dass sie sich zwar durch den Jobverlust neu orientieren konnte und nun viel besser über ihre Fähigkeiten, Interessen und Grenzen (bspw: nicht Vollzeit arbeiten zu können) Bescheid weiß, dies jedoch nicht als Stärke, sondern potenziell als „Arbeitsvereitelung " gesehen wird.

Nicht nur Z., sondern auch die anderen Mitglieder der Gruppe überlegen sich genau, ob und welche Stellen sie annehmen können. Das mag in Anbetracht der Einstellungspraxis in der Arbeitswelt, die ältere
Arbeitssuchende benachteiligt und nur wenige anständig bezahlte Vollzeitstellen zu bieten hat, widersinnig erscheinen, doch sie haben gute Gründe: Zum einen haben sie die Erfahrung des zunehmenden Leistungsdrucks in den letzten Arbeitsverhältnissen gemacht, die meist in (teils schwerwiegenden) gesundheitlichen Problemen endete. In dieses „Hamsterrad“ wollen sie nicht zurück. Zum anderen sind die meisten während der Erwerbsarbeitslosigkeit durchaus nicht beschäftigungslos, sondern widmen sich anderen Aufgaben (bpsw. Pflege der Eltern) oder Interessen (bpsw. ehrenamtliches Engagement, Weiterbildung). Die Wahl zwischen Burnout bzw. Hamsterrad und Boreout bzw. Standby entspricht damit nicht ihren Vorstellungen von einem „Leben, das man aus guten Gründen wertschätzt", wie es der Capability-Ansatz von Sen anstrebt. „Mich leben können“ schrieb Z. auf die Bitte hin auf, drei Elemente eines guten Lebens zu nennen. K. formulierte diesen Drang zur Selbstbestimmung als Wunsch „nie Würde und Visionen zu verlieren."

Von „Investitionen in den Menschen“ durch die aktive Arbeitsmarktpolitik haben die meisten Teilnehmenden wenig gespürt. Beim AMS hat $\mathrm{H}$. die Erfahrung gemacht, dass eine für ihn passende spezialisierte Schulung (in diesem Fall: Schulung in einer aktuellen Lagerverwaltungs-Software, wenn die Grundprinzipien der Lagerverwaltung bekannt sind) nicht bewilligt wurde, obwohl er nachweisen konnte, dass er dann eine Chance auf eine bestimmte Stelle gehabt hätte. K., die vor der Familiengründung und Anstellung im Betrieb ihres Mannes im Tourismus tätig war, hätte eine Schulung für die aktuell gebräuchlichen computergestützten Systeme gebraucht, um wieder einsteigen zu können. Dies wurde ihr jedoch verwehrt, weswegen sie auf prekäre Beschäftigungsverhältnisse zurückgeworfen wurde:

(...) ich hab jeden Gelegenheitsjob angenommen, den ich kriegen konnte. Und wo geht das? In der Gastro. Weil ich war da gerade 50 geworden. Ich war damals schon $z u$ alt für das meiste. Und für die meisten. (...) und hab dann einfach systematisch in der Gastro alles abgeklopft, was ging. Aber das sind immer nur limitierte Geschichten. Also, das waren Saisongeschichten etc. Und das blieb eigentlich die ganze Zeit so. Plus, ich hab Sozialhilfe beantragt, Mindestsicherung. Was bleibt dir denn übrig?"

B. dagegen konnte die Pensionsversicherungsanstalt überzeugen, ihm eine Umschulung zum Finanzbuchhalter zu finanzieren, da er seinen früheren Beruf in der Gastronomie nach einem Bandscheibenvorfall nicht mehr ausüben konnte und schon 93 Bewerbungen 
geschrieben hatte, ohne je zu einem Bewerbungsgespräch eingeladen worden zu sein. Bei der beruflichen Neuorientierung war er durch das Programm „Fit2Work“ begleitet worden, ein Berufseignungstest half ihm bei der Findung des neuen Berufswunsches. Nach anfänglichen Schwierigkeiten aufgrund fehlender Berufserfahrung („ein Titel ohne Mittel“, B.) hat er mittlerweile eine Stelle in einer öffentlichen Institution bekommen, schätzt dies jedoch eher als glücklichen Zufall denn als erwartbare Entwicklung ein.

Hier stellt sich die grundlegende Frage, wer solche Investitionen in Menschen finanzieren soll - die Wirtschaft, die Arbeitnehmerinnen und Arbeitnehmer oder die öffentliche Hand? H. musste jedenfalls auch über ein Jahr mit dem AMS streiten, um einen kurzen, günstigen Kurs zu bekommen, den er als wichtig ansah. „AMS - da brauche ich ja zwei Jahre, bis ich eine Schulung kriege!"

Zugleich haben die Teilnehmenden die Erfahrung gemacht, dass Fort- und Weiterbildungen bei ihren Bewerbungen kaum eine Rolle spielen: Von Arbeitgeberseite aus werden sie mit ihrer Erstausbildung identifiziert, auch wenn diese teils schon dreißig Jahre zurückliegt. Eine Neuorientierung ist unter diesen Umständen schwierig bis unmöglich. Weiterbildung wird nicht anerkannt (Lechner/Wetzel, 2015: 9). Selbst mit einer zweiten, gut abgeschlossenen und gesuchten Ausbildung ist es im fortgeschrittenen Alter schwierig, eine Stelle zu finden, solange die Berufserfahrung in diesem Bereich fehlt. B. musste zu einem unbezahlten Praktikum greifen, um die nötige Berufserfahrung zu erlangen. Mitglieder der Steuerungsgruppe, die schon länger in dem Bereich arbeiten, bestätigen, dass es heute meist sogar schwierig sei, ein Praktikum zu ergattern und Neuorientierungen für Ältere daher kaum möglich seien. Hinzu kommt, dass Praktika während des Bezugs von Arbeitslosengeld nur durchführbar sind, wenn das AMS dem zustimmt. Insofern werden Investitionen in ältere Erwerbspersonen von der Wirtschaft tendenziell nicht wertgeschätzt, geschweige denn forciert.

M. machte die Erfahrung von - unterschwelliger Altersdiskriminierung, als ihm in der AMS-Beratung von Umschulungen und Fortbildungen mit dem Hinweis: „Das steht Ihnen nur im Weg.“ abgeraten und somit implizit angedeutet wurde, dass eine solche Maßnahme als zwecklos angesehen wird. Einer Teilnehmerin wurde auf die Frage nach einer bestimmten Weiterbildung beschieden, sie müsse erst zwei bis drei Jahre arbeitslos sein, bevor das AMS bereit sei, Weiter- bildungen für sie zu fördern. Grundsätzlich gilt, dass den Teilnehmenden bisher nur in engen Grenzen eine Mitsprache bei der Auswahl der Weiterbildungsmaßnahmen gewährt wurde (Riesenfelder/Wetzel (2009) kommen zu etwas anderen Ergebnissen).

In ältere arbeitslose Menschen wird jedenfalls aus Sicht der Betroffenen nicht nur nicht investiert, sondern auch ihre eigenen Investitionen in Form von ehrenamtlichen Aktivitäten und Weiterbildungen werden durch den Status der Erwerbsarbeitslosigkeit eher untergraben als gestärkt und damit ihre Verwirklichungschancen eingeschränkt.

Gefordert werden von den Teilnehmenden mehr Mitspracherechte bei der Arbeitssuche, um individuelle Fähigkeiten und Bedürfnisse zu berücksichtigen, und Entscheidungsfreiheit bezüglich der Annahme oder Ablehnung qualitativ „schlechter“ Jobs. Sie streben also erstens nach relativen Autonomiegewinnen innerhalb der bestehenden Rahmenbedingungen. Perspektivisch fordern sie jedoch zweitens einen offensiven, investiven Ansatz von Seiten der Politik und vor allem auch der Wirtschaft, welcher Ältere selbstverständlich als Teil der Erwerbsarbeitswelt anerkennt und dementsprechende Rahmenbedingungen schafft und drittens eine kritische Auseinandersetzung mit der aktuell bestehenden Dominanz der Erwerbsarbeit für nahezu alle Dimensionen der Sozialintegration.

\subsection{Kontext: Reformen zur Beschäftigung Älterer}

Grundsätzlich ist die aktive Arbeitsmarktpolitik in Österreich vergleichsweise stark ausgebaut. In Folge der Wirtschaftskrise kam es zu keinen drastischen Ausgabenkürzungen, jedoch gingen die Aktivierungsausgaben pro Kopf aufgrund des Sparkurses in Folge der Krise weiter zurück und setzten damit einen schon einige Jahre zuvor einsetzenden Trend fort (Fink 2006, in: Hermann/Flecker, 2009: 35). Als Folge stehen nun weniger Mittel für Beratung, Schulung, Coaching und Beihilfen zur Verfügung. Die Priorität liegt auf rascher Wiedereingliederung durch Lohnsubventionen oder Platzierung auf dem erweiterten Arbeitsmarkt (kurier. at 2014). Zugleich wurden die Mittel der passiven Arbeitsmarktpolitik nach der schrittweisen Reduzierung und der Verschärfung der Zumutbarkeitsbestimmungen zwar nicht weiter gekürzt, aber auch nicht aufgestockt. Auch hier ist also das Muster eines schleichenden Prozesses, der durch die Wirtschaftskrise nur verstärkt wurde, erkennbar. 
Was die Situation älterer Erwerbspersonen betrifft, zeigt sich deutlich, dass „in Österreich ein höheres Alter ,per se die Beschäftigungschancen - im Sinn erhöhter Arbeitslosigkeitsquoten und einer verringerten Wahrscheinlichkeit einer raschen neuerlichen Beschäftigung - reduziert“ (Titelbach et al. 2015: 43). Es sind also nicht primär die Charakteristika der älteren Arbeitssuchenden ausschlaggebend. Allerdings besteht auch ein breiter öffentlicher Konsens, dass aufgrund der demografischen Entwicklung zur Finanzierung des Pensionssystems grundsätzlich eine Verlängerung des Arbeitslebens erforderlich ist.

Aufgrund dieser Widersprüchlichkeit kam es als Reaktion auf die Pensionsreformen zunächst zu beträchtlichen Verschiebungseffekten von einem System in das andere. Mittlerweile sind die meisten "Schlupflöcher“ geschlossen, die Arbeitsmarktchancen Älterer sind jedoch weiterhin schlecht. Ein Jobverlust in der „Endphase“ (welche jedoch leicht ein bis zwei Jahrzehnte umfassen kann) des Erwerbslebens ist deshalb unter den gegenwärtigen Bedingungen mit größeren Armuts- und Ausgrenzungsgefährdungen und dem Risiko eines enormen Statusverlustes verbunden.

Die Politik reagierte auf die steigende Erwerbsarbeitslosigkeit von älteren Erwerbspersonen mit einer Erhöhung der Mittel für Aktive Arbeitsmarktpolitik für die Zielgruppe 50+. Für die Jahre 2016 und 2017 wurde das Budget mehr als verdoppelt, wobei jedoch rund 60 \% fix für befristete Lohnsubventionen (Eingliederungsbeihilfe und Kombilohn) vorgesehen sind, ohne Möglichkeit der fallbezogenen Umwidmung von Mitteln für andere Maßnahmen. Langfristige und hochwertige Qualifizierungsmaßnahmen sind für ältere Arbeitssuchende (im Gegensatz zu anderen Zielgruppen, bspw. jungen Menschen) kaum verfügbar. Außerdem werden noch immer rund $8 \%$ des Gesamtbudgets für Aktive Arbeitsmarktpolitik für Leistungen, die auf den vorzeitigen Austritt aus dem Arbeitsmarkt abzielen (Altersteilzeit, Pensionsvorschuss, Übergangsgeld), aufgewendet (Bock-Schappelwein u. a. 2014: 83).

Die Arbeitsmarktpolitik für Ältere in Österreich folgt insofern keinen Social-Investment-Prinzipien, da sie kaum präventiv agiert und nur in einem sehr engen Verständnis Fähigkeitenstärkend wirkt.

\section{Schluss: Zählt wirklich nur die Arbeit?}

Dieser Beitrag ist ein Gemeinschaftswerk der Autorinnen mit den Teilnehmenden des Projekts RE-InVEST. Es ist die verschriftlichte Fassung eines
Vortrags zu den ersten Ergebnissen des Projekts in Salzburg, den wir gemeinsam gehalten haben. Kernbegriffe, Gliederung und Überschriften wurden gemeinsam entwickelt und bilden im Sinne des „Merging of Knowledge“ (Abschnitt 2) eine Synthese des aus der Literatur gewonnenen wissenschaftlichen Wissens wie auch des Erfahrungswissens der Betroffenen und der Sicht von Praktikerinnen und Praktikern aus dem Bereich. Durch diese Form der Wissensproduktion konnte wissenschaftlicher und praktisch-lebensweltlicher Erkenntnisgewinn zugleich erreicht werden. Die forschungsethischen Vorteile partizipativer Aktionsforschung wie Stärkung der Beteiligten und Schadensvermeidung sowie Sichtbarmachung und Teilhabe gesellschaftlich marginalisierter Gruppen und Individuen (Unger 2014: 11) sind klar erkennbar. Einerseits wurde mit den Aktionen die Sichtbarkeit der Gruppe und ihrer Probleme erhöht. Andererseits haben die Teilnehmenden bekundet, dass es sie gestärkt hat, über diese Themen reden zu können, $\mathrm{zu}$ merken, dass sie nicht allein sind und dass es nicht ihr persönliches Versagen ist, sondern strukturelle Gründe identifizierbar sind. Der Schritt an die Öffentlichkeit kostete sie große Überwindung, wurde jedoch als gewinnbringend hinsichtlich ihres Empfindens von gesellschaftlicher Partizipation und Teilhabe und als notwendige "Aktion“ für einen politischen und gesellschaftlichen Wandel beurteilt.

Der Prozess hat viele Erkenntnisse zutage gefördert: Dass die Krise von 2008 in Österreich kein einschneidendes Erlebnis war, dass die Teilnehmenden eine schleichende „Dehumanisierung der Arbeitswelt“ wahrnehmen, dass die erwerbslosen Betroffenen in Folge der Arbeitslosigkeit oft plötzlich viele Probleme zugleich lösen müssen und dabei wenig systematische Unterstützung erfahren, dass $\mathrm{zu}$ unterscheiden ist zwischen den Leitlinien der Arbeitsmarktpolitik und ihrer konkreten Umsetzung durch das AMS, dass die Arbeitssuchenden das AMS als „Blackbox“ erleben und nach einem „freien Fall“ im "Standby-Modus“ weiterleben. Die Arbeitsmarktpolitik und ihre konkrete Umsetzung durch das AMS werden in einer zweiten partizipativen Projektphase im Mittelpunkt stehen.

Schon jetzt steht jedoch ein zentrales Ergebnis der Arbeit fest: Derzeit kommt der Erwerbsarbeit eine zentrale Rolle zu, wenn es darum geht, wer zur österreichischen Gesellschaft „dazugehört“. Wer keine bezahlte Arbeit bzw. einen vergleichbaren akzeptieren Status hat, dem oder der wird die Anerkennung entzogen. Pflege und andere unbezahlte Arbeit werden 
nicht wertgeschätzt. Dies wirft Fragen für die gesamte österreichische Gesellschaft auf, denn in Anbetracht der krisenbedingten und längerfristigen Entwicklung auf dem Arbeitsmarkt und der weiteren Herausforderungen in Form der alternden Gesellschaft und der Digitalisierung der Arbeitswelt ist klar, dass Erwerbsarbeitslosigkeit jede und jeden treffen kann. Ist es nicht an der Zeit, sich zurückzubesinnen auf die Werte der Menschenrechtskonvention, darauf, dass Menschen zuerst Menschen sind und nicht Arbeitskräfte?

\section{Literatur}

Arbeiterkammer Wien (2014): Invaliditätspension Neu. $A K$ Aktuell. Das Infoservice der AK, Nr. 4/2014. https://media.arbeiterkammer.at/wien/AKAktuell_Nr_4_2014.pdf [28.06.2016].

Arbeitsmarktservice Österreich (2015a): Ältere am Arbeitsmarkt: Bedeutung der Generation 50+ steigt. Spezialthema zum Arbeitsmarkt. Online: http://www.ams. at/_docs/o01_spezialthema_0215.pdf [28.06.2016].

Arbeitsmarktservice Österreich (2015b): Langzeitarbeitslosigkeit und Langzeitbeschäftigungslosigkeit. Spezialthema zum Arbeitsmarkt. Online: http://www.ams. at/_docs/oo1_spezialthema_1115.pdf [01.07.2016].

Arbeitsmarktservice Österreich (2015c): Alter hat Zukunft. Informationen für ältere Arbeitssuchende (45+). Informationsbroschüre des Arbeitsmarktservice Österreich. Online: http://www.ams.at/_docs/oor_alterhatzukunft. pdf [01.07.2016].

Basso, G./Dolls, M/Eichhorst, W./Leoni T./Peichl, A. (2011): The Effects of the Recent Economic Crisis on Social Protection and Labour Market Arrangements across Socio-Economic Groups. Nr. 608o. Discussion Paper Series. IZA - Forschungsinstitut zur Zukunft der Arbeit.

Bednarek-Gilland, A. (2015): Fragiler Alltag: Studie zu den Fähigkeiten langzeitarbeitsloser Menschen. Hannover: creo-media.

Bergold, J/Thomas, S. (2012): Partizipative Forschungsmethoden: Ein methodischer Ansatz in Bewegung. Forum Qualitative Sozialforschung, 13 (1), Art. 30. Online: http:// www.qualitative-research.net/index.php/fqs/article/ view/1801/3332 [05.01.2017].

BMASK (Bundesministerium für Arbeit, Soziales und Konsumentenschutz) (2014): Sozialbericht 2013-2014. Ressortaktivitäten und Sozialpolitische Analysen. Wien: Bundesministerium für Arbeit, Soziales und Konsumentenschutz.

Bock-Schappelwein, J./Fuchs, S./Huemer, U./Mahringer, H./Konle-Seidl, R./Rhein, T. (2014): Aktive und Passive Arbeitsmarktpolitik in Österreich und Deutschland. Aufkommen und Verwendung der Mittel im Vergleich. Wien: Österreichisches Institut für Wirtschaftsforschung. Online: http://www.wifo.ac.at/jart/prj3/wifo/ resources/person_dokument/person_dokument. jart?publikationsid $=47166 \&$ mime_type $=$ application $/$ pdf [28.06.2016].

Burchardt, T./Vizard, P. (2011): ,Operationalizing the Capability Approach as a Basis for Equality and Human Rights Monitoring in Twenty-first-century Britain. Journal of Human Development and Capabilities 12 (1), 91-119.

derStandard.at (2015): Massiver Anstieg bei AMS-Beschwerden. derStandard.at, 21.01.2015. Online: http://derstandard.at/2000010637352/VolksanwaltAMS-Beschwerden-beim-stiegen-massiv [28.06.2016].

Elson, D. (Hg.) (2014): Human rights and the capabilities approach: an interdisciplinary dialogue. London: Routledge.

Egger-Subotitsch, A./Poschalko, A./Kerler, M./Bartok, L. (2014): Optimale Betreuung und Vorgangsweise bei psychisch belasteten KundInnen. Forschungsprojekt im Auftrag des AMS Burgenland. Wien: abif - analyse beratung interdisziplinäre forschung.

Fink, M. (2006): Zwischen „Beschäftigungsrekord“ und „Rekordarbeitslosigkeit“: Arbeitsmarkt und Arbeitsmarktpolitik unter Schwarz-Blau/Orange. In: Tálos, E. (Hg.): Schwarz-Blau. Eine Bilanz des "Neu-Regierens“. Reihe Politik und Zeitgeschichte, Band 3. Münster u.a.: LIT Verlag, 170-187.

Fink, M. (2015a): ESPN Thematic Report on Social Investment Austria. Bericht an die Europäische Kommission im Rahmen des European Social Policy Networks. Wien. Online: http://ec.europa.eu/social/BlobServlet?docId=1 3808\&langId $=$ en [28.06.2016].

Fink, M. (2015b): ESPN Thematic Report on Integrated Support for the Long-Term Unemployed Austria. Bericht an die Europäische Kommission im Rahmen des European Social Policy Networks. Wien: Institut für Höhere Studien. Online: http://ec.europa.eu/social/BlobServlet?docI $\mathrm{d}=14244$ \&langId $=$ en [28.06.2016].

Graf, G. (2015): Capability Approach. In: Nida-Rümelin, J./ Spiegel, I./Tiedemann, M. (Hg.): Handbuch Philosophie und Ethik. Band 2: Disziplinen und Themen. Paderborn: UTB, 312-317.

Hanappi, T./Müllbacher, S./Heil, E. (2014): Auswirkungen von finanziellen Anreizen auf das Pensionsantrittsalter. Eine Analyse mit dem Mikrosimulationsmodell IREA. Studie im Auftrag des Bundesministeriums für Finanzen. Wien: Institut für Höhere Studien. Online: https://www.ihs. ac.at/publications/lib/IHSPR6501143.pdf [28.06.2016].

Hermann, C./Flecker, J. (Hg.) (2009): Die Dynamik des „österreichischen Modells". Brüche und Kontinuitäten im Beschäftigungs- und Sozialsystem. Berlin: edition sigma.

Hermann, C./Flecker, J. (2014): Die Krise bewältigt, aber die Zukunft nicht. Das österreichische Modell in der Finanz- und Wirtschaftskrise. In: Lehndorff, S. (Hg.): Spaltende Integration: der Triumph gescheiterter Ideen in Europa - revisited: zehn Länderstudien. Hamburg: VSA, $175-187$.

Jahoda, M./Lazarsfeld, P. F./Zeisel, H. (2014): Die Arbeitslosen von Marienthal: ein soziographischer Versuch über die Wirkungen langandauernder Arbeitslosigkeit; mit einem 
Anh. zur Geschichte der Soziographie. 24. Aufl. Edition Suhrkamp 769. Frankfurt am Main: Suhrkamp.

Kraemer, K. (2009): Prekarisierung - Jenseits von Klasse und Stand? In: Castell, R./Dörre, K. (Hg.): Prekarität, Abstieg, Ausgrenzung: Die soziale Frage am Beginn des 21. Jahrhunderts. Frankfurt am Main: Campus, 241-52.

kurier.at. (2014): Stark steigende Arbeitslosigkeit führt zu Budgetsorgen beim AMS. Online: http://kurier.at/wirtschaft/ stark-steigende-arbeitslosigkeit-fuehrt-zu-budgetsorgen-beim-ams/85.791.058 [28.06.2016].

Land Salzburg, Abteilung Soziales (2015): Sozialbericht 2013/2014. Jahresbericht. Salzburg. Online: http:// landversand.salzburg.gv.at/WebRoot/Store/Shops/ Landversand/55C9/B 977/C8Fo/D3 $\mathrm{FE} / \mathrm{ABDD} / 4 \mathrm{DEB} /$ $\mathrm{AE}_{3} \mathrm{E} / 3 \mathrm{ACB} /$ Sozialbericht2014-Internet.pdf [11.01.2017].

Lechner, F./Wetzel, P. (2015): EEPO Review Spring 2015: Upskilling Unemployed Adults The Organisation, Profiling and Targeting of Training Provision - Austria. Bericht an die Europäische Kommission im Rahmen des European Employment Policy Observatory (EEPO). L\&R Social Research OG. Online: http://ec.europa.eu/social/BlobSe rvlet?docId $=14145$ \&langId $=$ en [28.06.2016].

Mayring, P. (1993): Qualitative Inhaltsanalyse. Grundlagen und Techniken. Weinheim: Deutscher StudienVerlag.

Murphy, M./Hearne, R. (2015): RE-InVEST Methodological Toolkit, unveröffentlichtes Manuskript, RE-InVESTPapers.

Nussbaum, M. (1999): Gerechtigkeit oder Das Gute Leben. Hg. von Herlinde Pauer-Studer. Frankfurt am Main: Suhrkamp.

Nussbaum, M. (2002): Capabilities and Human Rights. In: de Greiff, P./ Cronin, C. (Hg.): Global Justice and Transnational Policies. Cambridge, Mass.: MIT Press, 117-150.

orf.at. (2013): Neues Landesbudget steht. 18.10.2013. Online: http://salzburg.orf.at/news/stories/2610031/ [28.06.2016].

orf.at. (2015): Starker Rückgang bei AMS-Schulungen. 2.02.2015. Online: http://orf.at/stories/2263883/2263588/ [28.06.2016].

orf.at. (2016): Bankenrettung kostete Österreich neun bis 14 Mrd. Euro. 27.10.2016. Online: http://orf.at/stories/236400o/ [27.01.2017].

Riesenfelder, A./Wetzel, P. (2009): Evaluierung von AMSKursen und deren Effekte auf einschlägige Beschäftigung und Einkommen. Endbericht des Projekts. Wien. Online: http://www.lrsocialresearch.at/files/EB_Eval_Kurse_ AMS_L\&R_Sozialforschung.pdf [28.06.2016].

Robeyns, I. (2005): The capability approach: a theoretical survey. Journal of Human Development, 6 (1), 93-117.

Sedmak, C./Gaisbauer, H. P./Gstach, I./Rohrauer, M./Schweiger, G. (2014): Studien- und Evaluierungsarbeit mit Beteiligungs- und Dialogprozess zur Entwicklung eines Maßnahmenkonzepts zur Inklusion von arbeitsfähigen, aber nicht beschäftigungsfähigen Menschen. Studie im Auftrag des Landes Salzburg. Salzburg: Zentrum für Ethik und Armutsforschung der Universität Salzburg/internatio- nales forschungszentrum für soziale und ethische fragen.

Sen, A. (2003): Ökonomie für den Menschen. Wege zu Gerechtigkeit und Solidarität in der Marktwirtschaft. München: Deutscher Taschenbuch Verlag.

Sen, A. (2005): Human Rights and Capabilities. Journal of Human Development, 6 (2), 151-166. Online: https://doi. org/10.1080/14649880500120491.

Sen, A. (2010): Die Idee der Gerechtigkeit. München: C. H. Beck.

Statistik Austria (2015): Öffentliches Defizit. Online: http:// www.statistik.at/web_de/statistiken/wirtschaft/oeffentliche_finanzen_und_steuern/maastricht-indikatoren/ oeffentliches_defizit/o19649.html [28.06.2016].

Statistik Austria (2016): Erwerbstätigenquoten nach Alter und Geschlecht seit 1994. Online: http://www.statistik.at/ $\mathrm{wcm} /$ idc/idcplg? IdcService=GET_PDF_FILE\&Revision SelectionMethod $=$ LatestReleased $\&$ dDocName $=062876$ [28.06.2016].

Titelbach, G./Müllbacher, S./Fink, M./Hofer, H. (2015): Arbeitslosigkeitsbetroffenheit und Wiederbeschäftigung Älterer im Vergleich zu Jüngeren am österreichischen Arbeitsmarkt. Endbericht - Studie im Auftrag des Sozialministeriums. Wien: Institut für Höhere Studien. Online: http://irihs.ihs.ac.at/3664/1/IHSPR6741174.pdf [28.06.2016].

von Unger, H. (2014): Partizipative Forschung. Einführung in die Forschungspraxis. Wiesbaden: Springer VS.

WKO (Wirtschaftskammer Österreich) (o.J.): Freier Dienstvertrag (sozialrechtlich). Online: https://www.wko.at/ Content.Node/Service/Arbeitsrecht-und-Sozialrecht/ Arbeitsrecht/Beschaeftigungsformen/Freier_Dienstvertrag_(sozialrechtlich).html [29.06.2016]. 